\title{
AN ONTOLOGY DRIVEN APPROACH TO SOFTWARE SYSTEMS COMPOSITION
}

\author{
A Thesis \\ Presented to \\ The Faculty of Graduate Studies \\ of \\ The University of Guelph \\ by \\ HLOMANI HLOMANI \\ In partial fulfilment of requirements \\ for the degree of \\ Master of Science
}

July, 2009

(C) Hlomani Hlomani, 2009 


$\begin{array}{ll}\begin{array}{l}\text { Library and Archives } \\ \text { Canada }\end{array} & \begin{array}{l}\text { Bibliothèque et } \\ \text { Archives Canada }\end{array} \\ \begin{array}{l}\text { Published Heritage } \\ \text { Branch }\end{array} & \begin{array}{l}\text { Direction du } \\ \text { Patrimoine de l'édition }\end{array} \\ \begin{array}{l}\text { 395 Wellington Street } \\ \text { Ottawa ON K1A 0N4 } \\ \text { Canada }\end{array} & \begin{array}{l}\text { 395, rue Wellington } \\ \text { Ottawa ON K1A ON4 } \\ \text { Canada }\end{array}\end{array}$

Your file Votre reférence

ISBN: 978-0-494-56731-9

Our file Notre reférence

ISBN: 978-0-494-56731-9

\section{NOTICE:}

The author has granted a nonexclusive license allowing Library and Archives Canada to reproduce, publish, archive, preserve, conserve, communicate to the public by telecommunication or on the Internet, loan, distribute and sell theses worldwide, for commercial or noncommercial purposes, in microform, paper, electronic and/or any other formats.

The author retains copyright ownership and moral rights in this thesis. Neither the thesis nor substantial extracts from it may be printed or otherwise reproduced without the author's permission.

\section{AVIS:}

L'auteur a accordé une licence non exclusive permettant à la Bibliothèque et Archives Canada de reproduire, publier, archiver, sauvegarder, conserver, transmettre au public par télécommunication ou par l'Internet, prêter, distribuer et vendre des thèses partout dans le monde, à des fins commerciales ou autres, sur support microforme, papier, électronique et/ou autres formats.

L'auteur conserve la propriété du droit d'auteur et des droits moraux qui protège cette thèse. $\mathrm{Ni}$ la thèse ni des extraits substantiels de celle-ci ne doivent être imprimés ou autrement reproduits sans son autorisation.
In compliance with the Canadian Privacy Act some supporting forms may have been removed from this thesis.

While these forms may be included in the document page count, their removal does not represent any loss of content from the thesis.
Conformément à la loi canadienne sur la protection de la vie privée, quelques formulaires secondaires ont été enlevés de cette thèse.

Bien que ces formulaires aient inclus dans la pagination, il n'y aura aucun contenu manquant.

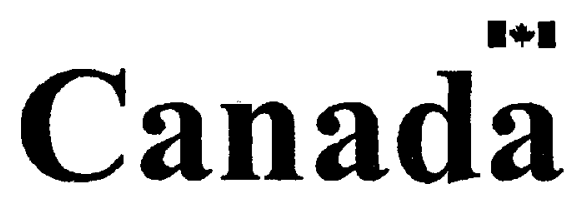




\begin{abstract}
AN ONTOLOGY DRIVEN APPROACH TO SOFTWARE SYSTEMS COMPOSITION
\end{abstract}

Hlomani Hlomani
University of Guelph, 2009
Advisors:

Dr. D.A. Stacey

Since its inception the semantic web has been gaining interest and acceptance in researchers from different domains leading to the development of tools, standards and technologies. The development of Ontologies (semantic web technologies) can be adapted to serve software development methodologies. Thus the goal of this research is to demonstrate that the use of ontologies for system composition delivers a user-centric and flexible system making this approach suitable for dynamic domains. This is demonstrated through a proof of concept prototype system driven by knowledge embodied in a set of Ontologies:an algorithm Ontology and an execution timeline Ontology. The main idea behind Ontology Driven Compositional System (ODCS) is allowing domain experts to compose a system by choosing the system components and the desired interactions between these components in a way suitable to their problem. 


\section{Acknowledgments}

I would like to take this opportunity to thank my supervisor Deborah Stacey for the time, effort and all the help she offered during the course of this work. I am truly grateful to her for her willingness to sit with me and help explain concepts that I did not understand and for the overall guidance during the development and writing of this thesis.

My heartfelt gratitude also goes to the Botswana International University of Science and Technology for making this dream come true by providing the funding for my studies. This would not have been possible if it were not for their initiative. A warm thank you also goes to my family and all those who sacrificed two years of living without me around, your pátience and words of encouragement are greatly appreciated. Thank you once again to all who helped me in one way or the other and to all those who made my stay here in Canada a pleasant time. 


\section{Table of Contents}

List of Figures $\quad$ iv

List of Tables vi

1 Introduction 1

1.1 Motivation . . . . . . . . . . . . . . . . . . . 1

1.2 Thesis Statement . . . . . . . . . . . . . . . . . . . 2

1.3 Contributions . . . . . . . . . . . . . . . . . . . . 3

1.4 Organization of the Thesis . . . . . . . . . . . . . 4

2 Literature Review $\mathbf{5}$

2.1 Semantic Web ........................... 5

2.1 .1 Ontologies . . . . . . . . . . . . . . . 7

2.1.2 Application of Ontologies: A Syndromic Surveillance Example 7

2.2 Other Approaches to Systems Composition . . . . . . . . . . . . . . . 17

2.2 .1 Web Services . . . . . . . . . . . . . . 17

3 Our Approach and the Proposed Prototype 19

3.1 The System Architecture . . . . . . . . . . . . . . . . . . . . 20

3.2 The Ontologies . . . . . . . . . . . . . . . . . . . . . 20

3.2.1 The Algorithms Ontology . . . . . . . . . . . . . 22

3.2 .2 The Execution Timeline Ontology . . . . . . . . . . . . . 31

3.3 The Access Class Libraries . . . . . . . . . . . . . . . . . . . . 32

3.3.1 Generation of Class Library . . . . . . . . . . . . . . 35

3.3.2 OWL to Java Mismatches and their Fixes . . . . . . . . . 37

3.4 Application Logic . . . . . . . . . . . . . . . . . . . . . . 38

3.4 .1 Overview of the System's Functionality . . . . . . . . . . 38

3.4.2 Application Logic Framework . . . . . . . . . . . . . . . . . 42

3.5 Composing a System and Ontology Manipulation . . . . . . . . . . . 44

3.5.1 Ideal Method of System Construction . . . . . . . . . . . . 45

3.5 .2 Current Setup . . . . . . . . . . . . . . . 45

3.6 Assumptions . . . . . . . . . . . . . . . . . . . . . . . . . . . . . . . . 48

3.7 Summary . . . . . . . . . . . . . . . . . . . . . . . . . . . . . . 49 
4 Case Studies $\mathbf{5 1}$

4.1 Background and Purpose . . . . . . . . . . . . . . . . 51

4.1 .1 System Composition . . . . . . . . . . . . . . 52

4.1.2 The Architectures and the Possibility of Extension . . . . . . 52

4.1.3 Software Quality Attributes: Flexibility, Reusability, and Testability . . . . . . . . . . . . . . . 55

4.2 The Case Studies . . . . . . . . . . . . . . . . . . . . 58

4.2 .1 The Algorithms . . . . . . . . . . . . . . . . . . . . . . . . . 59

4.2 .2 The Data . . . . . . . . . . . . . . . . . . 63

4.2.3 Case Study 1: Concurrent Running of Algorithms . . . . . . . 63

4.2.4 Case Study 2: Voting . . . . . . . . . . . . . 67

4.2.5 Case Study 3: Automated Use of the Architectural Elements . 70

4.2 .6 Concurrency test . . . . . . . . . . . . . . 73

5 Analysis $\quad 75$

5.1 Reusability and the Aspects of the Approach that

Addresses it . . . . . . . . . . . . . . . . 76

5.2 Flexibility and the Aspects of the Approach that

Addresses it . . . . . . . . . . . . . . . . . . 80

5.3 Testability . . . . . . . . . . . . . . . . . . . . 85

5.4 Limitations of the Approach . . . . . . . . . . . . . . . . . 86

5.4 .1 Critical Mass . . . . . . . . . . . . . . . . . . 86

5.4 .2 Trust . . . . . . . . . . . . . . . . . 86

5.4 .3 Performance . . . . . . . . . . . . . . . 87

6 Conclusions and Future Work $\quad 89$

6.1 Conclusions . . . . . . . . . . . . . . . . . . . . . . . . . . . 89

6.2 Future Work . . . . . . . . . . . . . . . . . . . . . . . 91

6.2.1 Population of the Algorithm Ontology . . . . . . . . . . . . 91

6.2 .2 Ontology Evolution . . . . . . . . . . . . . . . . . 91

6.2.3 Integration of Existing Ontologies . . . . . . . . . . . . . 92

6.2.4 Architectures and Design Patterns" . . . . . . . . . . . . 92

6.2 .5 Graphical User Interface . . . . . . . . . . . . . . . . . . . . 93

6.2 .6 Verification and Validation . . . . . . . . . . . . . . . 94

$\begin{array}{ll}\text { A Overview of the Ontologies } & 96\end{array}$

A.1 Overview of the Algorithm Ontology . . . . . . . . . . . . . 96

A.2 Overview of the Execution Timeline Ontology . . . . . . . . . . 99

$\begin{array}{ll}\text { Bibliography } & 100\end{array}$ 


\section{List of Figures}

2.1 Overview of BIOSTORM's Architecture and Components . . . . . . 11

2.2 BioCaster system components . . . . . . . . . . . . . . . . . 14

2.3 Generic Human Disease Ontology . . . . . . . . . . . . . . . . 17

3.1 The Architecture . . . . . . . . . . . . . . . . . . . . . 21

3.2 Overview of the algorithm Ontology's major classes and their relationships . . . . . . . . . . . . . . . . . . . 24

3.3 Properties of the AlgorithmFile class and its subclasses . . . . . . . 25

3.4 Properties of the OperatingEnvironment class and its subclasses . . . 26

3.5 Properties of the algorithm class and its subclasses . . . . . . . . . 27

3.6 Properties of the Input class and its subclasses . . . . . . . . . . . . 29

3.7 Properties of the Output class and its subclasses . . . . . . . . . . 30

3.8 Properties of the Execution Timeline Ontology . . . . . . . . . . . . . 32

3.9 A Java example of the Algorithm Class Interface . . . . . . . . . . . 34

3.10 A Java example implementation of the Algorithm Class Interface. . . 35

3.11 An example usage of the Algorithm Class . . . . . . . . . . . . . . . 36

3.12 Overview of a Pipe and Filters style . . . . . . . . . . . . . . . . . 39

3.13 Overview of a vote design . . . . . . . . . . . . . . . 40

3.14 Overview of a pipe and filters style with another element providing a bridging function ....................... 41

3.15 Overview of a bridged vote design . . . . . . . . . . . . . 41

3.16 An example TaskGraph and the extraction of Events. . . . . . . . . . 44

3.17 Example Test Class: Creation of Tasks . . . . . . . . . . . . 46

4.1 Overview of McCall's Software Quality Factors . . . . . . . . . . . . 58

4.2 Algorithm class hierarchy(Java Classes) . . . . . . . . . . . . . . . 60

4.3 Overview of case study 1 timeline . . . . . . . . . . . . . . 66

4.4 Overview of case study 1.1 timeline . . . . . . . . . . . . 67

4.5 Overview of a plot of the results of the algorithms in the case study 1.1 timeline . . . . . . . . . . . . . . . . 68

4.6 Overview of case study 2 timeline . . . . . . . . . . 70

4.7 Demonstration of the use of the architectural elements . . . . . . 71

5.1 Overview of a possible branch arrangement . . . . . . . 84

A.1 Overview of the Thing Class Hierarchy (immediate children) . . . . 96

A.2 Overview of the AlgorithmFile Class Hierarchy . . . . . . . . . . . 96 
A.3 Overview of the Algorithm Class Hierarchy . . . . . . . . . . . . . . 97

A.4 Overview of the Input Class Hierarchy . . . . . . . . . . . . . . . 97

A.5 Overview of the OperationEnvironment Class Hierarchy . . . . . . . 98

A.6 Overview of the Output Class Hierarchy . . . . . . . . . . . . . 98

A.7 Overview of the ExecutionTimeline Ontology Class Hierarchy . . . . 99 


\section{List of Tables}

4.1 The created events in case study $1 \ldots \ldots . \ldots 65$

4.2 The created events in case study $1.1 \ldots \ldots \ldots 6$. . . . . . . . . 69

4.3 The created events in case study $2 \ldots \ldots$. . . . . . . . . . . 69

4.4 The created events in the demonstration of usage of architectural elements ........................ . . 72

4.5 Concurrency times with the actual Syndromic Surveillance algorithms (Case Study 1) . . . . . . . . . . . . . . . . 73

4.6 Concurrency with dummy programs that have delays on them . . . 73 


\section{Chapter 1}

\section{Introduction}

\subsection{Motivation}

A 'modular' approach to systems engineering is not a new concept but one that deserves further research and interest. In this approach a system is decomposed into smaller parts, the parts are developed individually and a new system is built by composing these parts into a well balanced composite system. This approach to system development enables certain attractive traits including reusability, flexibility and encapsulation of implementation details.

From a developer's perspective reusability is an important concept in his/her daily job. From a user's perspective, however, flexibility and control over the application may be paramount. The developer also has the huge task of shielding the user from the overwhelming implementation details by encapsulating these technical details and only exposing to the user the functions they need.

The premise of this thesis therefore is that no single solution exists for all problems at hand as some domains are faced with very dynamic problems dictated by many parameters and environmental settings. This implies the need for systems that can be rapidly developed and changed to fit a given task. The changes may be in 
altering the constituent components (e.g. algorithms) or in altering the performance and functionality of these algorithms. The problem though is that with current system development approaches, modifying the system would require a substantial amount of technical proficiency and time. In most disciplines (e.g. syndromic surveillance, also know as disease detection, monitoring, and real-time situational awareness) it may be desirable to have the very users of the system (e.g. epidemiologists) dictate, among other things, the algorithms suitable for a problem, the appropriate data sources, and the arrangement and flow of data between the constituent algorithms. These users may be technically naïve and, therefore, it may be desirable for these users (domain experts) to not concern themselves with the programming aspect of composing the system. We therefore propose a prototype system that has at its core several Ontologies that provide rich descriptions of the components of a system thereby allowing for the autonomous creation and modification of systems which are problem or mission oriented by the very domain experts who need to use them.

\subsection{Thesis Statement}

An Ontology driven compositional system has flexibility, reusability, and testability quality attributes making it suitable for dynamic application areas that require a user-centric and rapid application development. This will be demonstrated

by a review of a selection of case studies that illustrate these attributes of a prototype system that follows this approach.

User-centric is used in the context of having the users being the ones doing 
the system development. These users are domain experts who specialize in a specific area (e.g. Epidemiology, Economics) and may or may not have any software development skills. Dynamic application areas are domains that are characterized by the use of experimental systems, research oriented, and use of concepts that are outside their domain (e.g. algorithms) in performing specific tasks.

\subsection{Contributions}

The development and use of Ontologies has gained popularity and hence there is a growing collection of Ontologies in existence. However Ontologies for algorithms are very rare. We introduced an Ontology to describe algorithms that serve as the fundamental elements of a compositional system. This Ontology is different in that its description focuses primarily on describing what is needed to run an instance of an algorithm. This Ontology also plays a pivotal role in system composition as it is an enabler of the interconnection of algorithms by providing descriptions of architectural elements that affects the flow of data within a composed system.

Another contribution of this research is an Ontology to describe the overall order of event execution. This is particularly important because it exposes what happens within the system in a format that is somewhat intuitive to the user. It offers flexibility in tailoring and ordering the constituent algorithms to suit a specific need.

A prototype system that uses these Ontologies as descriptors of its component was developed. While this is not an exhaustive system, it serves the purpose 
of demonstrating the concept of a compositional system that uses Ontologies as descriptors of the base components of the system.

\subsection{Organization of the Thesis}

The chapter that follows discusses two fields of interest; semantic web and syndromic surveillance. It explores the characteristics of these fields and their underlying technologies with the view of determining the suitability of the semantic web to a composition system and the characteristics that make syndromic surveillance a suitable candidate application area for the proposed prototype system. Chapter 3 outlines the approach followed in the development of the prototype system. It discusses the components of this system including Ontologies, class libraries and major decisions that had to be taken. Case studies that illustrates aspects of this system are discussed in chapter 4 . Chapter 5 provides reflections and discussions of the case studies and the power afforded by this approach. We conclude this thesis by: presenting our deductions, conclusions and thoughts on future work in chapter 6 . 


\section{Chapter 2}

\section{Literature Review}

In exploring the concept of compositional systems two fields of interest are considered; the semantic web and syndromic surveillance. The semantic web is considered because of its ability to provide descriptions of system components through the use of Ontologies thereby enabling system components to be composed and put together for a common goal. Syndromic surveillance is considered as an example application area. This literature review examines these two fields. It looks at the characteristics of these fields and their underlying technologies with the view of determining the suitability of the semantic web to a composition system and the characteristics that make syndromic surveillance a suitable candidate application area for the proposed prototype system.

\subsection{Semantic Web}

The semantic web is defined by Shadbolt, Hall and Berners-Lee as a web of data as opposed to the current web which is a web of documents [31]. It is envisioned to extend the current web by giving web content semantic meaning for the betterment of cooperation between computers and humans [10]. The semantic web can also be 
seen as a technology by which information is presented in both natural language and machine-interpretable format aiding in sharing and integration of information [42]. Central to the semantic web approach is the use of Ontologies. These are formal computer stored specifications of concepts in a domain and their relationships to provide for a common platform for information exchange [38].

The promise of the semantic web has fueled interest from researchers in many different domains [16]. This has led to many applications, tools and technologies being developed based on its concept of producing and consuming machine-processable information. One of these technologies is the Ontology authoring languages [16]. The other improvement within the semantic web area is the approval of specifications like the Resource Description Framework (RDF) and the Web Ontology Language (OWL) $[16,31,42]$ : RDF is the foundation for processing meta-data. It provides interoperability between applications that exchange machine-interpretable data over . the web $[10]$. OWL on the other hand is a standard Ontology language from the World Wide Web Consortium (W3C) used to specify concepts and their relationships [38]. Research in the semantic web field has also led to further research and development of tools and plug-ins that aid in development of semantic web components. One major tool used is the Protégé Integrated Development Environment (IDE)[27]. Protégé has àlso been extended to produce Protégé-OWL which supports OWL [35]. 


\subsubsection{Ontologies}

As mentioned earlier Ontologies play a pivotal role not only in the advancement of the semantic web but in knowledge sharing and management at large. The use of Ontologies is a principle that was coined from the field of philosophy to provide a common description of concepts. This use of Ontologies is one of the things that bring the semantic web dream within reach. Even though the definition of Ontologies across literature is not a concise one, most authors have managed to highlight the main aspects of Ontologies in their definition. They have been defined in [38] as formal computer stored specifications of concepts in a domain and their relationships to provide for a common platform for information exchange. Zhang [42] provides a not so concise definition of Ontology. He asserts that an Ontology is a rich expression of semantic relations. The definition is, however, vague and misses some of the important aspects provided in [38] above. Another definition that follows the one provided in [38] is the one provided in [16] where Ontologies are defined as descriptions of concepts in a domain and the relationships that holds between those concepts.

\subsubsection{Application of Ontologies: A Syndromic Surveillance Example}

Some domains have managed to harness and take advantage of the potential of Ontologies. One of these fields is the field of Syndromic Surveillance. Even in well disciplined and highly structured domains like the medical field that has well established taxonomies and highly valued coding systems disparities do occur. These can be avoided with the use of semantic web technologies. A trivial example is given 
in [25], it states that semantic web technologies can be used to infer that the terms "fever", "pyrexia" and "elevated temperature". all refer to the same concept even though they are represented differently by coding systems such as SNOMED. Further investigation can be performed to infer other concepts such as the description of fever, its cause and semantic relations between established terms and synonymous terms that are used to refer to it. Therefore these existing semantic relations allow automation of processes such as syndrome definition, aberration and outbreak detection. In this section we will highlight the characteristics of the syndromic surveillance field and discuss how the semantic web has been used to address issues pertaining to syndromic surveillance.

The overall objective of Syndromic Surveillance is early identification of outbreak occurrences to avoid fatalities [14]. Syndromic Surveillance has been defined as the monitoring of pre-diagnostic health-related data for early detection of outbreaks [7]. Another definition of syndromic surveillance that agrees with the above assertions is presented by Sosin [33]. The author, however, uses the term public health surveillance instead of syndromic surveillance which when read in the context used in the article is synonymous with syndromic surveillance. It is defined as an "ongoing, systematic collection, analysis and interpretation of health-related data needed for the planning, implementation and evaluation of the public health practices". All of these definitions and most of the other definitions not mentioned in the this thesis revolve around the acquisition of data, the analysis of that data using detection algorithms, with the purpose of early detection of outbreaks. 
Even though Syndromic Surveillance does offer the ability to prevent the widespread of diseases and outbreaks, the process is rather intricate. Syndromic surveillance relies heavily on the acquisition, integration and analysis of health-related data from various data sources. Moreover, the application of various algorithms to the data for early detection of disease outbreaks is also crucial. Surveillance data source are heterogenous and the data itself very spatial and temporal intensive. This renders the data highly dimensional and therefore posing a big challenge of integrating the data sources into surveillance systems [7]. These disparate data sources must be integrated into the system in a manner that offers semantic coherence and assists in decision making for outbreak detection.

Syndromic surveillance is a data intensive process, in fact Crubezy et al [7] attributes the proliferation of syndromic surveillance systems to the increased availability of electronic data. They however, contend that this increase in availability of electronic data does not only present a vast number of opportunities to the syndromic surveillance system but rather along with these opportunities come several challenges. For timely and efficient surveillance, syndromic surveillance systems have to analyze data from several physically heterogeneous data sources: whose data are often represented in different formats, at different granularity and often contain a lot of noise. They also assert that these challenges are further compounded by the fact that outbreak patterns are also unpredictable and dynamic in nature. These problems render the conventionally developed systems with predefined data sets and analytical methods unsuitable for syndromic surveillance. Their proposition is that 
a surveillance system should have a range of analytical methods suitably chosen and tailored to each situation at hand. This is in contrast to the traditional setup that has a set of predefined algorithms that are configured to specific data sets. This implies a setup where both data sources and analytical methods are rapidly included and excluded from the system as the situation dictates.

The semantic web has shown some promise in addressing the above mentioned challenges. This was demonstrated by a semantic web enabled prototype system discussed and developed by the same authors called Biological Spatio-Temporal Outbreak Reasoning Module (BIOSTORM) [7]. BIOSTORM is a system that integrates distinct data sources and employs various analytical methods with the aim of supporting health specialist to interpret surveillance data and detect outbreaks. As is with many semantic web enabled systems, central to BIOSTORM is the use of Ontologies to model and annotate syndromic surveillance resources. Ontologies in the system are used to provide descriptions of the properties, types, relationships of the data and also to provide description of the problem solvers employed in the system. The system's four main components are depicted by Figure 2.1.

The components of the system include:

- A data source Ontology that describes and harmonizes the data sources and their disparate semantics.

- A library of problem solvers that work on the surveillance data to detect disease outbreaks.

- A mediation component which consists of two subcomponents 


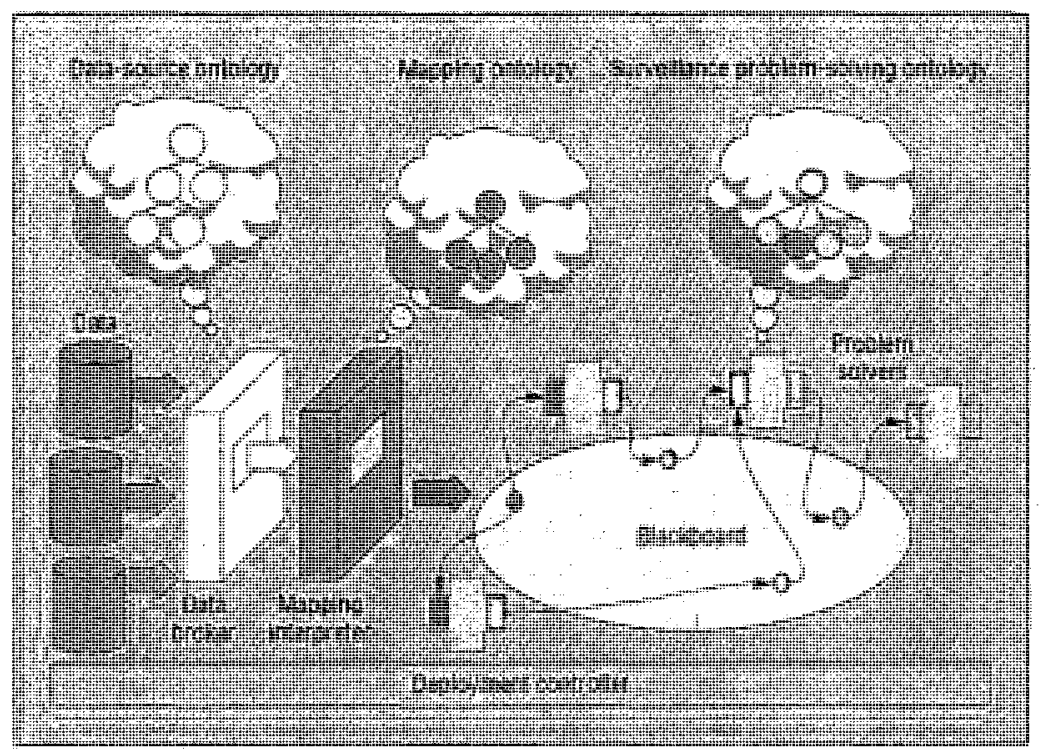

Figure 2.1: Overview of BIOSTORM's Architecture and Components (source: [7])

- A data broker which does the actual integration of multiple related data sources described by the data source Ontology

- Mapping interpreter which connects the integrated data sources to those problem solvers that can best analyze that particular data

- and last but not least a controller which identifies and deploys relevant configurations for the problem solver to analyze the input data.

Three Ontologies are used in the BIOSTORM system namely, data source Ontology, a data mapping Ontology and a problem solving Ontology. With the first two being used for the acquisition of data from various data sources and the third is to include and apply problem solvers to the preprocessed data sets.

The BIOSTORM team's sentiments are also shared by [6], who in his masters 
thesis claims that current surveillance systems do have the necessary facets needed to provide specific solutions to specific problems. He, however, contends that these systems fall short in providing extensibility to allow for new data sources and inclusion of new problem solvers. Chruszcz attributes this limitation to system design choices that places heávy reliance on experts with knowledge and the technical ability to make even the slightest of changes. His propositions are somewhat related to the BIOSTORM solution. He advocates for the addition of a description layer to a syndromic surveillance system that makes knowledge explicit at every level of the system thus exposing the system functions and processes to the user. This explicit definition of knowledge is achieved by the use of Ontologies. These abilities were demonstrated by a Semantic Web Assisted Syndromic Surveillance System (SWASS) whose main components are Ontologies that provide descriptions for the input, the algorithms and the output of the system.

Chruszcz [6] asserts that the BIOSTORM team has contributed to the advancement of syndromic surveillance by applying semantic web technologies, the author, however, contends that their work was incomplete. The team according to Chruszcz did extensive work in describing and providing facilities for the inclusion of different data sources into one system but less work was done in providing descriptions of the algorithms that does the actual analysis of the data and on the final output of the system. These are the extensions that are provided in SWASS.

Ontologies make the semantic web highly suitable for syndromic surveillance since they provide rich descriptions of the underlying components of the system. 
This facilitates the work of unifying the data sources that are normally disjoint, integrating them into the system and mapping them to the relevant problem solvers. Ontologies enable these functionalities while retaining the semantics or meanings of the underlying data.

A majority of syndromic surveillance systems rely on their traditional public health partners like emergency departments, hospitals and laboratories making them their primary source of data [26]. This means that in most cases syndromic surveillance systems will have emergency department logs, over-the-counter pharmaceutical sales, job and school absenteeism, and tele-health data as their data sources $[12,26]$. However, there are other unconventional potential data sources for syndromic surveillance which include monitoring video cameras in public places to measure incidences of coughing and keeping record of orange juice sales from grocery stores [26]. Some of the data sources that generate interest and have the potential of increasing the rapid detection of outbreaks are news articles and informal information source available on the web. Kawazoe et al [21] estimates that $65 \%$ of initial reports of outbreaks come from these data sources. Sifting through this large pool of information and information resources would however require an overwhelming amount of time and effort therefore some form of automation and computer based support would be in order. This came in the form of a text mining-based system called BioCaster discussed in [21]. A major component of this system is an event Ontology that describes event concepts and is the basis for machine understanding of the disease outbreak information. This event Ontology formally referred to as the BioCaster Event Ontology 


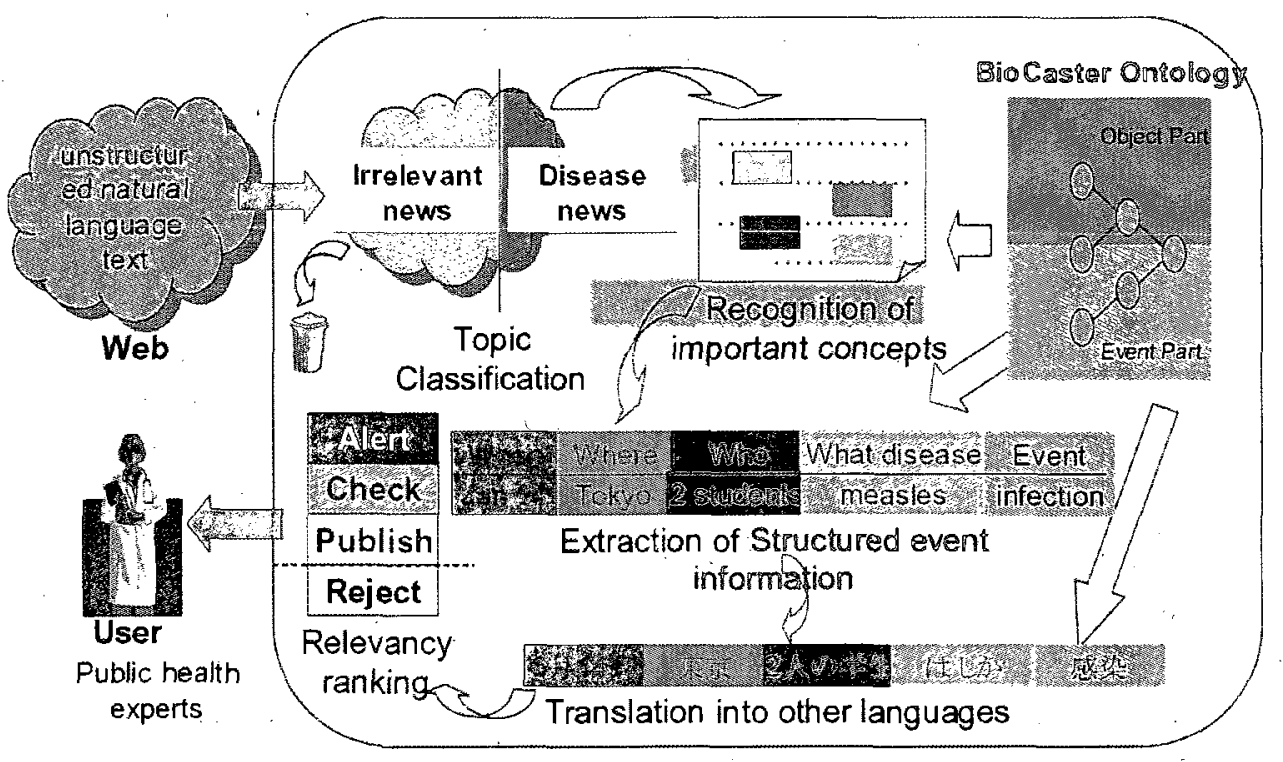

Figure 2.2: BioCaster system components (source: [21])

is used to define disease-related events such as newly emerging infectious diseases, abnormal cases of unknown outbreaks and drug resistant illnesses.

The main aim of the Ontology is to address two main issues: 1)Bridge the gap between layman's language and public health experts' knowledge. Reports on disease outbreaks contained in newspapers are often written in non-technical language while health specialists analyze outbreaks events with highly technical knowledge. It is therefore necessary to represent the information in a way that conforms to their knowledge; 2)Multiple Linguistics. Kawazoe et al also assert that usually information on disease outbreaks is reported in local languages therefore raising the need to translate this foreign language into the experts' natural language. The BioCaster system is summed up by figure 2.2 . 
With the current web setup and the exponentially increasing availability of information on the Internet, it is becoming increasingly difficult to provide information that is relevant. Traditional search engines that are keyword reliant are often good enough to retrieve results but do not really account for the ambiguity in terminology usage that exists in different domains $[8,9]$. To do away with ambiguity several Ontologies have been developed in the biomedical field each having its own scope and purpose. These Ontologies provide explicit definitions of the terms within a domain of discourse, the relationships that hold between them and their properties. In this section we will discuss some of these Ontologies and how they are of assistance to syndromic surveillance and the outbreak detection course.

An exploration of how the semantic web technologies may be employed to bridge the gap created by ambiguity was done in the implementation of a National Library of Infection (NeLI) [8]. At the heart of this implementation is once again the use of Ontologies to provide a shared understanding of the domain (in this case Infection Domain). Diallo et al's work [8], however, focuses more on the description of the building process of the Ontology rather than the benefits of the Ontology itself. They do, however, enumerate the range of the vocabulary and concepts defined by the Ontology to include clinical microbiology, infectious diseases, infection control, public health, surveillance and the affected population. Definition of these concepts promotes a common understanding, reusability and interpretability. In syndromic surveillance a possible usage of this Ontology could be in the determination of a disease outbreak profile covering the description of the disease, its cause, etc. 
The work discussed above fairly relates to [13] whose main application domain for their Ontology is human disease research and control. This has much resemblance to syndromic surveillance since it deals with investigation and control of diseases. According to them, this is a domain that is usually characterized by high-quality, extremely complex, very heterogeneous information resources and its distributed nature. An important role that Ontologies play therefore is in unifying the resource allowing researchers to look at all different factors causing a human disorder simultaneously and identify possible links. The work described in their work is largely on the principles of building a Generic Human Disease Ontology which describes common general information concerning human diseases. The Ontology they describe in their work has four main parts namely: types - which describe the different types of the disease; phenotype - describing the symptoms of the disease; causes - describing the responsible causes of that specific disorder; treatment - describes possible treatments of a disease and overall efficiency of the treatment. As mentioned earlier this is a general Ontology that can further be specialized to derive other more specific Ontologies. The Generic Human Disease Ontology is depicted in figure 2.3. An interesting aspect about Ontologies is that they are extensible, making them highly suitable for dynamic domains like the biomedical domain which is evolving. As an example the Ontology discussed above can be extended by expanding the causes subclass to include a new disease cause that may have been realized after the initial construction of the Ontology. 


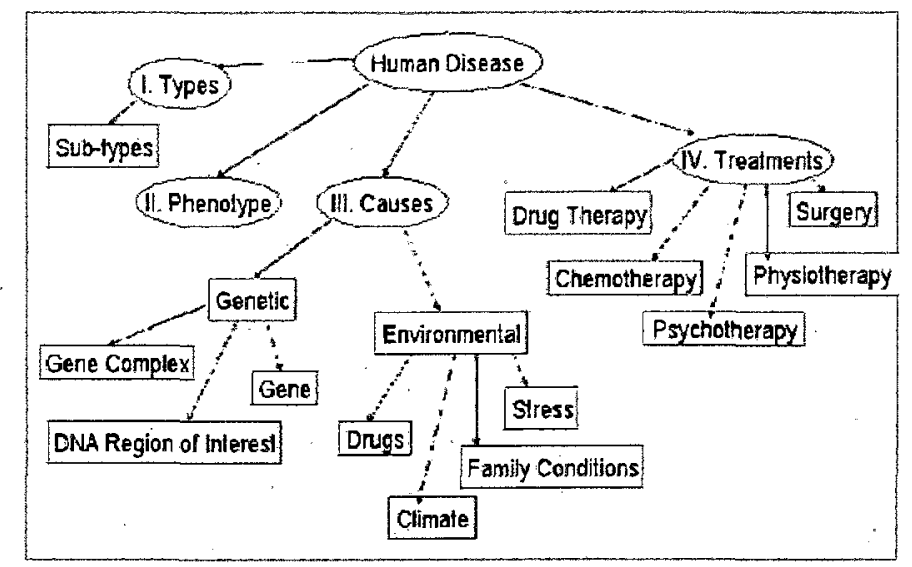

Figure 2.3: Generic Human Disease Ontology (source: [13])

\subsection{Other Approaches to Systems Composition}

\subsubsection{Web Services}

Web services are Service Oriented Computing (SOC) implementations that provide a standardized way of presenting some functionality in the form of integrated web-based applications using XML, SOAP, WSDL, UDDI and other internet protocols. They are distributed applications which can be discovered, bound to and interactively accessed in an autonomous manner [5]. They have also been defined in the literature as networked capabilities with openly accessible interfaces for other machines to discover and invoke in real time $[1,24]$. Web Services are network, technology and platform independent [29] making them highly interoperable. They are also loosely coupled making the idea of reusability very attainable.

Web Services fit into this discussion of system composition in that a functional system can be developed from putting together already existing web services 
in what is termed as service composition. Service composition is a cost effective approach which allows a developer to build a fully fledged application by using already existing components in the form of web services [24]. Although there may be some benefit in invoking a single web service, combining several already existing services and ordering them to best suit your requirements may bring added value [5, 24]. Developers can therefore use service composition to rapidly develop applications that meet users needs, meet an organization's goal, or provide a new service function $[24,11]$.

While service composition has advantages in theory, the adoption and use of discovery protocols and the lack of development of libraries of freely available and discoverable services has left this approach mostly unrealized. Since web services are by definition distributed and thus require distributed resources, this restricts their applicability in some domains where privacy and security are critical. It also necessitates that the user be constantly connected to the Internet. While the compositional system being proposed in this paper does not necessarily use web services, the utilization of distributed programs and resources is a logical extension of our system definitions and thus our system is a superset of the domain of all services/programs including web services. 


\section{Chapter 3}

\section{Our Approach and the Proposed Prototype}

Ontology development can be adapted to serve software development methodologies and vice versa [15]. Therefore, the primary focus of this thesis is to examine the characteristics and the suitability of using Ontologies as the main driver for a compositional system both for its development and post development functionality. By compositional system we mean a system that allows its components to be put together in a systematic manner to achieve a common goal or to derive yet another functional application. To demonstrate the plausibility of such a system, the Ontology Driven Compositional System (ODCS) was developed. This is a prototype system developed using the Java programming language guided by the use of Ontologies. This was done by building on current research in the use of semantic webs for application development. These include design patterns/architectures, tools and technologies.

The following sections will provide a discussion on the adopted system architecture that shows the components of the system and how they, all fit together. This will be followed by discussions of the individual system components including

the Ontologies used, generated class libraries and the system model or application 
logic. It is also imperative to provide a discussion on the major assumptions made during the development.

\subsection{The System Architecture}

An Ontology driven approach to software systems development is similar to other software development paradigms in that there has to be a guiding architecture that specifies the components within the intended systems and how these components relate to each other. For the semantic web a fitting architecture was presented by Knublauch [22]. The author differentiates between two layers of a semantic web application. First, there is a semantic web layer that hard-codes knowledge about a particular domain which is then used to model the behavior of the application. Second there is an internal layer which is composed of the reasoning mechanisms and the application's control logic that controls the application's functionality and interacts with the user via an interface. The prototype system discussed in this thesis follows this general architecture but, however, provides extended definitions of certain concepts particularly in the application logic. This architecture is depicted by Figure 3.1. In the discussions of the subsections that follow, several alternative solutions may be presented and a rationale for the choices made will be provided.

\subsection{The Ontologies}

The use of Ontologies to provide a domain specific knowledge base in order to facilitate for communication and knowledge sharing between people and various 


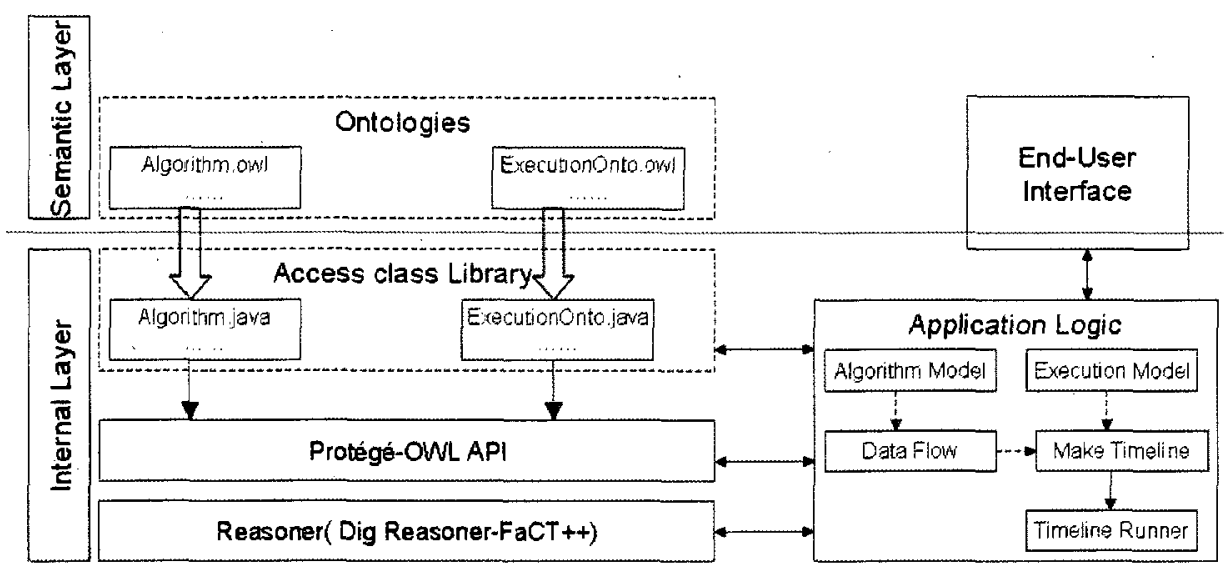

Figure 3.1: The Architecture (source: [22])

computer agents is arguably the pillar on which the semantic web hinges. Their benefits have been well enumerated and discussed across literature and therefore this implementation adds on to the non-ending list of demonstrations of the functionality of Ontologies. We however take it beyond just facilitating for knowledge sharing. We use this common knowledge to drive the development of a compositional system by using the Ontologies to provide an extensible description of the components of a system and how they can be put together. To achieve this we have created an Ontology to describe the algorithms that provide the needed functionality, data converters that provides conversion from a source data type to needed data type and other algorithmic elements that are deemed to be important to a compositional system. We have also defined an Ontology that describes the order of event execution within a composed system.

Throughout the thesis we have used the terms Algorithm and Program interchangeably, however, it is important to note the difference between the two. The 
former is a step-by-step plan for solving a problem often characterized by a finite number of steps that take in input and yield output $[40,41]$. While the latter is an expression of the former in a specific programming language such that a computer can execute it. Simply put; a program is an implementation of an algorithm in a certain programming language. In essence what is being described by the algorithm Ontology is the program, which in some cases may be an implementation of a single algorithm and in other cases the program may be implementing a collection of algorithms.

\subsubsection{The Algorithms Ontology}

This Ontology was designed to provide an extensible standard description of algorithms in the context of the elements or aspects of the algorithm that one would need to know about the algorithm in order to use the algorithm. The use of a common description of algorithms would allow for easier addition and removal of algorithms from a system or application as this will be done at the semantic level rather than at the programming level therefore reducing dependence on skilled personnel.

The development of an Ontology for algorithms, however, proved to be more complex than initially perceived. This can be attributed to the existence of diverse views and perceptions on classification of algorithms. Unlike in other domains where agreed upon taxonomies and classifications already exist, the same cannot be said about algorithms. Algorithms that follow a specific model or design paradigm can be grouped together to form a hierarchy. For example, Spears [34] and Langdon

[23] discuss Evolutionary Algorithms (EA) which are a subset of the Probabilistic 
and Heuristic paradigm. Their model is based on simulating evolution of individual through such concepts as selection, recombination and a mutation. Furthermore, EA will itself have sub-types under it two of which are Genetic Algorithms (GAs) and Evolutionary Programming (EP). While these are similar in nature they are different in their implementation and representation. Contrast this classification though with the view of grouping algorithms according to their purpose or goals ( e.g. sort algorithm, mathematical algorithms, graphical algorithms etc). Furthermore, another classification of algorithms could be based on the principle of how they are implemented ( e.g. recursive algorithms, deterministic algorithms, parallel algorithms etc.). Other algorithms may, however, span classes. Hence we cannot claim absolute correctness as different levels of abstractions and perceptions exists. However, it seems more reasonable to classify algorithms according to the reason they were designed (purpose). The focus of this Ontology, however, is not to provide a classification of algorithms but rather we focus on providing a generic description of the algorithms in order to facilitate the automation of the algorithms usage and provide a plug and play kind of functionality. Such elements as the algorithms operating environment, its input, output, and the algorithms type will be described.

The classes in the Ontology describe what is needed to run an instance of an algorithm defined by the subclasses of the Algorithm class. In this Ontology everything is a subclass of Thing including Algorithm which can be seen as the overarching concept. The algorithm concept is the very thing that all the other classes in the Ontology describe and relate to. As mentioned earlier the purpose of 


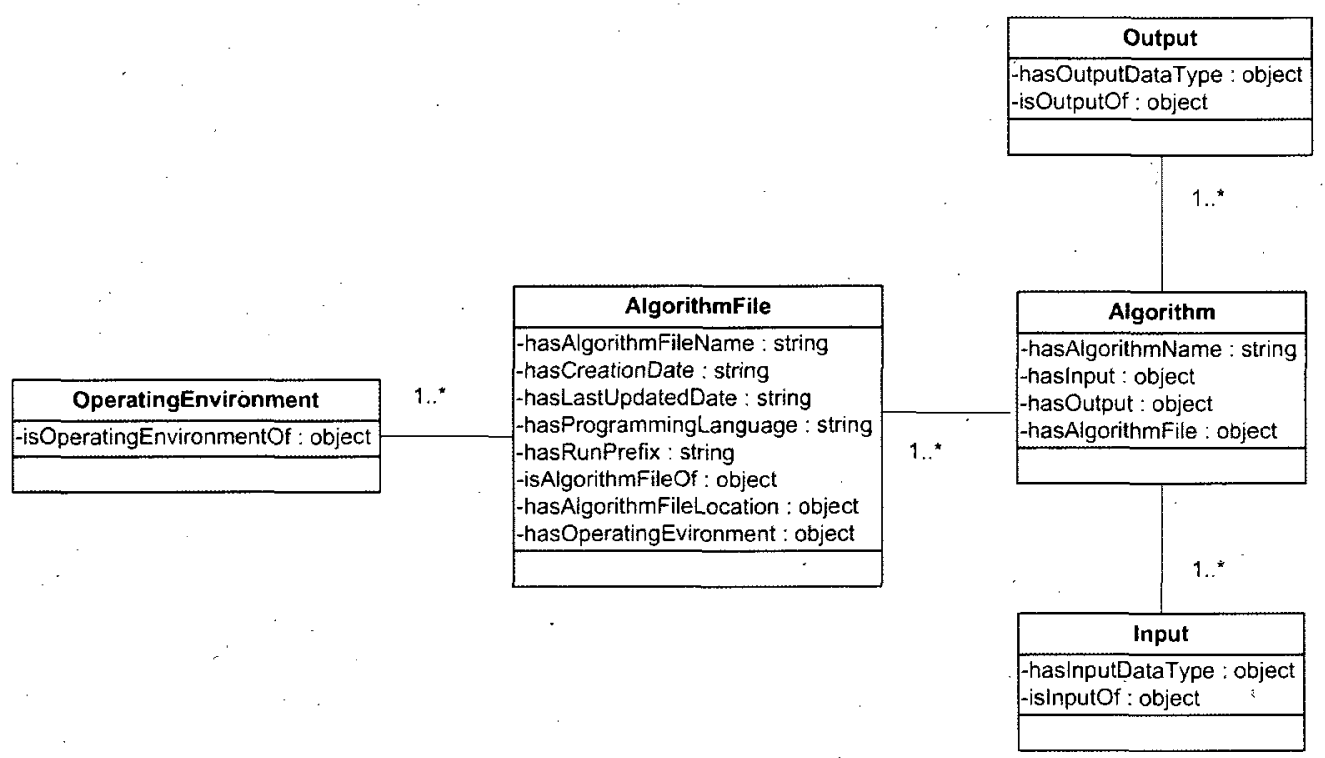

Figure 3.2: Overview of the algorithm Ontology's major classes and their relationships

this Ontology is not to devise a hierarchical classification of algorithms but rather describe the aspects of an algorithm. Figure 3.2 depicts some of the major classes of the algorithm Ontology and the relationships that holds between them. These classes are further described in subsequent paragraphs.

An algorithm. exists in some sort of file be it in its source code form or as a ready to run executable file. For this purpose we created a AlgorithmFile class which aims at describing aspects relating to the actual algorithm file. It defines such properties as the file name, creation date, last updated date, etc. (refer to Figure 3.3). It also defines object properties that provide linkage to location and operating environment classes. Each algorithm executable or source code file has a specific environment in which it can operate on. For this purpose we have an OperationEnvironment class that describes this environment. This include subclasses to describe the operating 


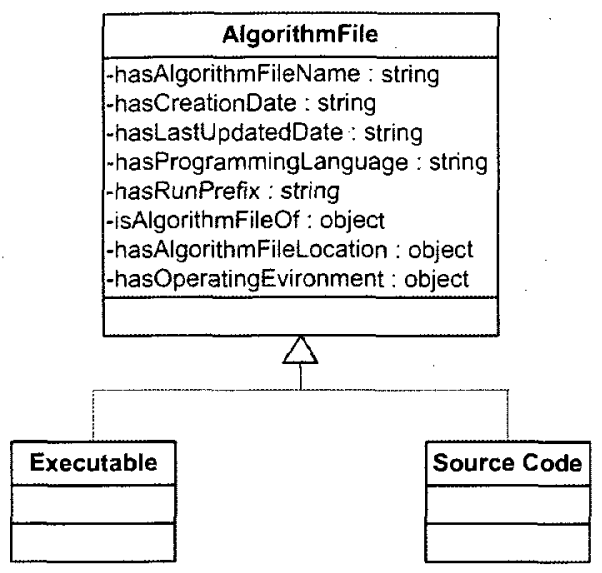

Figure 3.3: Properties of the AlgorithmFile class and its subclasses

system, drivers, and other software needed to run the algorithm as depicted by Figure 3.4 .

We differentiate between two main classes of algorithms: Computational Algorithms and Architectural Elements. We perceive computational algorithms to be the very algorithms that provide domain specific or general purpose functionality. Architectural elements are those pieces of code whose operation provide support to the computational elements and help in creating and maintaining the structure of the system. Specific subclasses can be defined under each of these classes to describe a needed type. Subclasses describe specific types of algorithms and define other properties that are relevant to that specific type. Figure 3.5 depicts this kind of hierarchical structure of the class. It is important to note that the current Ontology is not well populated with algorithms that are suitable for all domains and problems. It is, however, an example useful to the syndromic surveillance domain. If the system has to search for a statistical algorithm the results could be an instance of any of the subclasses of the 


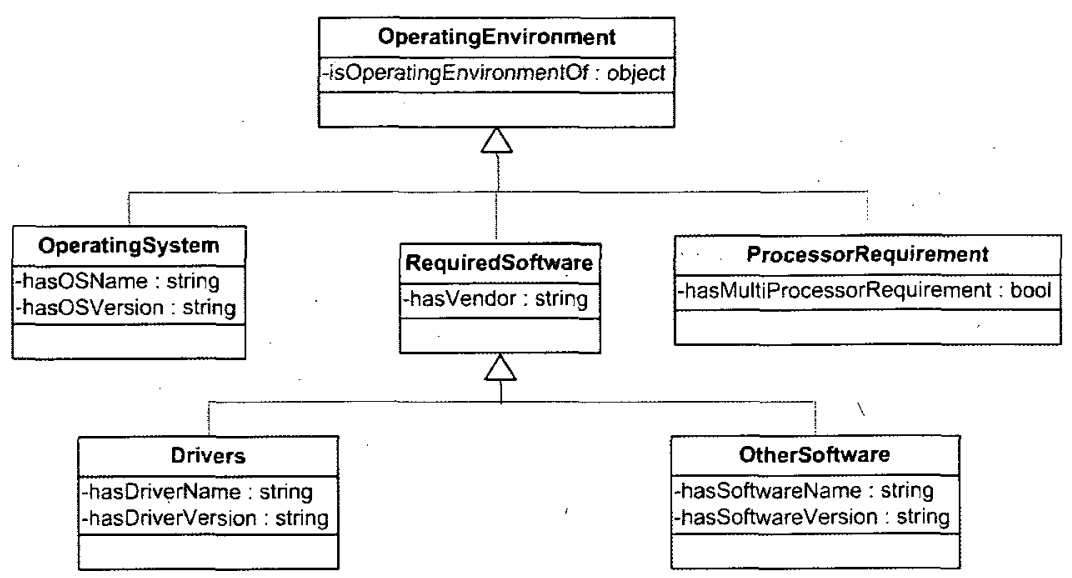

Figure 3.4: Properties of the OperatingEnvironment class and its subclasses

statistical algorithms e.g. an instance of an EMAAlgorithm (ExponentialMovingAverageAlgorithm) class since its instance is an instance of StatisticalAlgorithm due to inheritance. This inheritance structure allows the class to be extended when a new algorithm type definition is needed.

Most algorithms take in some sort of input, therefore for every instance of an algorithm there must be an indication of whether it receives any input and a precise definition of how the input is received. The Input class provides these descriptions. Input to an algorithm may be in the form of data files read by the algorithm, command line parameters passed through the input stream to the algorithm or read from configuration files. These are described by further defining subclasses of the Input class. For example, a Parameters class is defined and its subclasses are then defined to include the CommandLine class that describes the parameters passed through the input stream to the algorithm and Configurationfiles class that describes parameters read from configuration files. These subclasses can also be further subclassed 


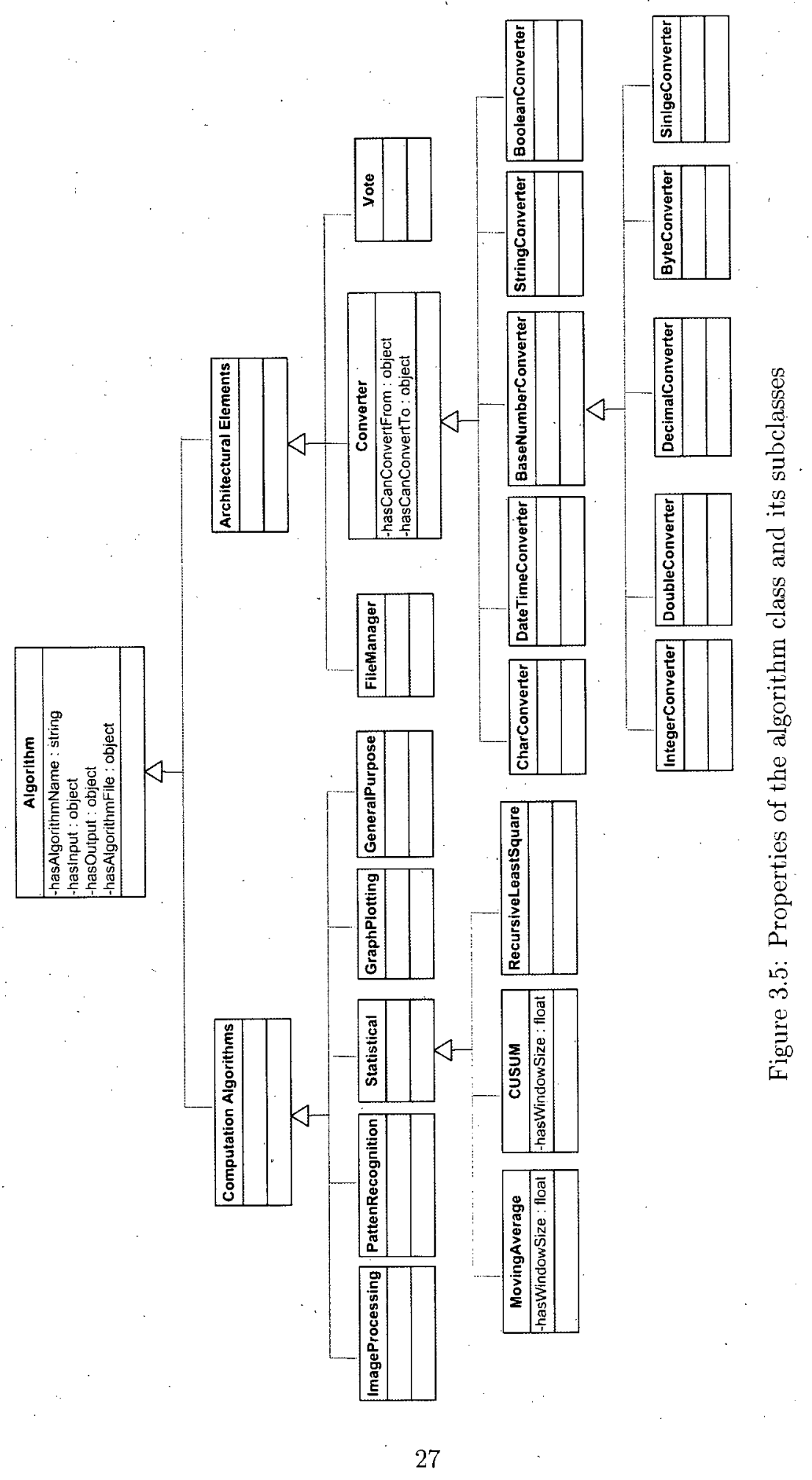


to provide needed details. This is depicted by Figure 3.6.

As a result of an álgorithm's processing, output is produced therefore the Ontology also needs to describe algorithm output. The Input, Output and a DataType class discussed below play a significant role in a compositional system. This is a system that relies heavily on connecting algorithms together for example piping one algorithm's output into another algorithms. Determining the compatibility of the algorithms is only possible when the input, output of an algorithm is known and the data types thereof. An algorithm's output can be a pipe through the output stream, some sort of multimedia output or output to a text file. These can be described by providing subclasses of the Output class for each of the possible output subtypes. This structure is depicted by Figure 3.7 . We have defined a DataType class to provide the description of the data type of each of the input and output of an algorithm instance. We acknowledge the existence of various ways of modeling this however for the purpose of this demonstration the DataType class has several instances each representing the possible data types an input or output can have. These could be primitive data types like boolean, string, and integer but could include complex data types as long as they are well defined within the Ontology. We have also defined a generic Location class. This class relates to several other classes within the Ontology to define the location of specific files needed for an algorithm instance to be run. Examples would include location details of an input database file or text file and location details of the actual algorithm executable or source file.

When composing a system by combining algorithms together through their 


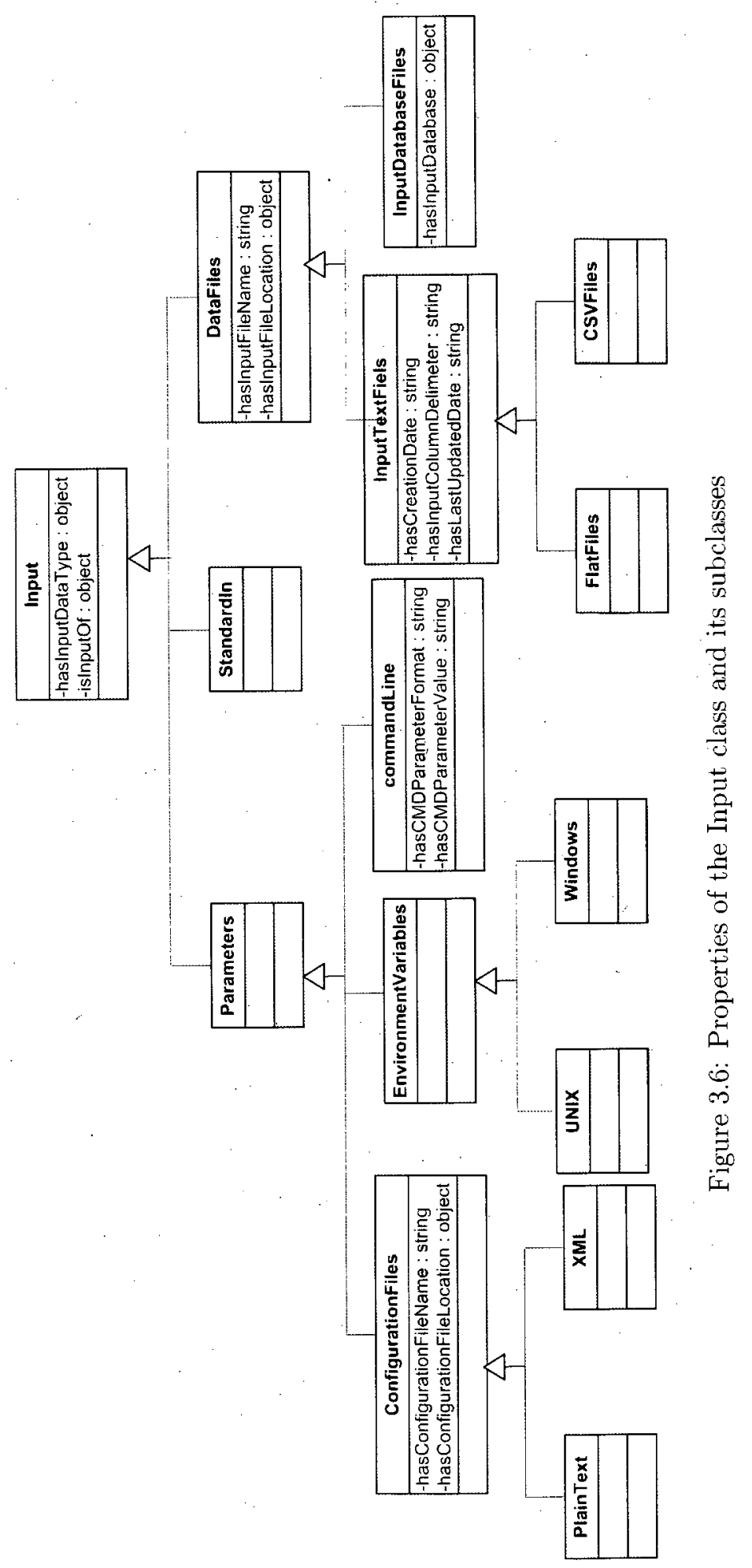




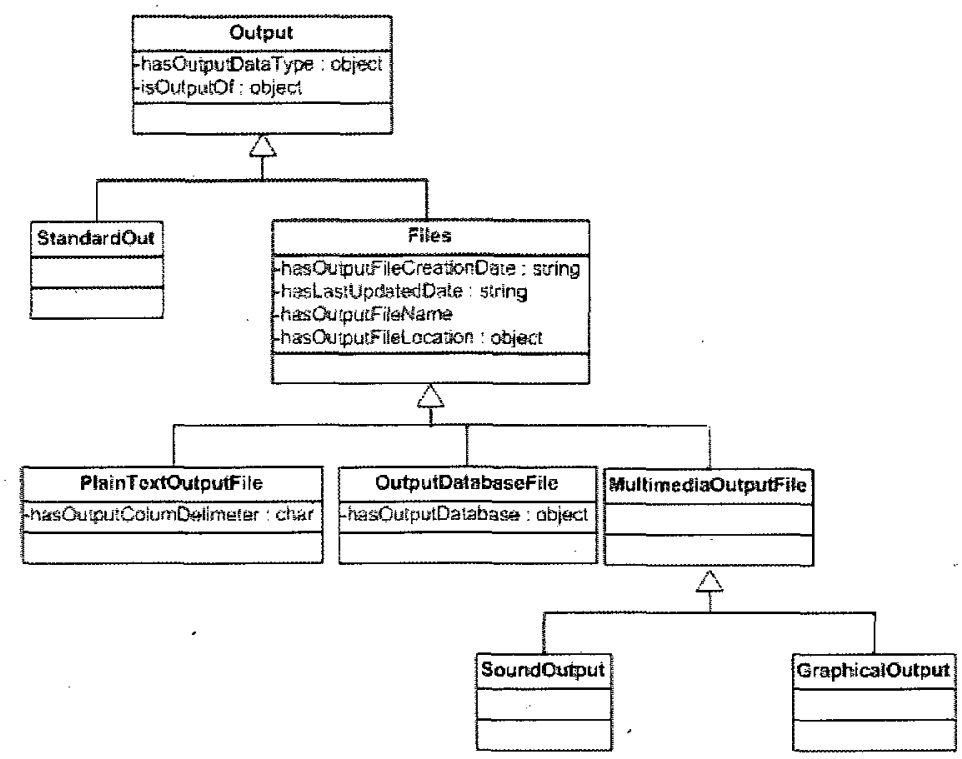

Figure 3.7: Properties of the Output class and its subclasses

input streams and output stream several incompatibilities may exist; these include data type mismatches and data stream mismatches. The architectural elements class (Figure 3.5) within the algorithms Ontology therefore serves to provide a generic description of these internal glue elements of the system. Data type converters are components of a compositional system that offers data conversion to facilitate compatibility between algorithms. Converters are algorithms in that they are standalone pieces of code that can be fed input and run to produce some output. An example mismatch is that of different data stream types feeding into and out of a pipe. One algorithm may be printing output to the standard stream while the algorithm it connects to expects input from a file. Therefore rather than just declaring the connection to be impossible an architectural element would be placed between these two algorithms to satisfy both algorithms' requirements. In this case the element would 
be reading data from the standard stream and writing it to a file so that the next algorithm can read the file.

\subsubsection{The Execution Timeline Ontology}

Ultimately the composed system will be run. To facilitate flexibility and extensibility we have developed an Ontology that describes how the events within the composed system will be run. Please refer to section 3.4 for detailed discussion . on the individual elements of a timeline. The basic functionality of this Ontology is to describe the order in which the connected algorithms will be executed. The Ontology defines this order in the form of a Timeline class that has a number of intervals. The Interval class has a collection of start and end triggers and a number of events. The Event class also has begin and end triggers. Specific triggers are defined as subclasses of the Trigger class. Triggers could for example be performing the function of checking for preconditions and postcondition of an event or an interval or specifying what the next process (event or interval) to run is. The Ontology also defines exit points within each event, interval and timeline in the form of errors handlers. A Unified Modeling Language (UML) representation of the classes and properties in this Ontology is depicted by Figure 3.8 .

Having decomposed and described the system into the aforemention ontological concepts presents the potential for several desirable traits. Events can be run concurrently and branching from one set of events to another can also be achieved allowing for flexibility in the functionality and structure of the application. Monitoring of events and the state of the application is also made possible since all events are 


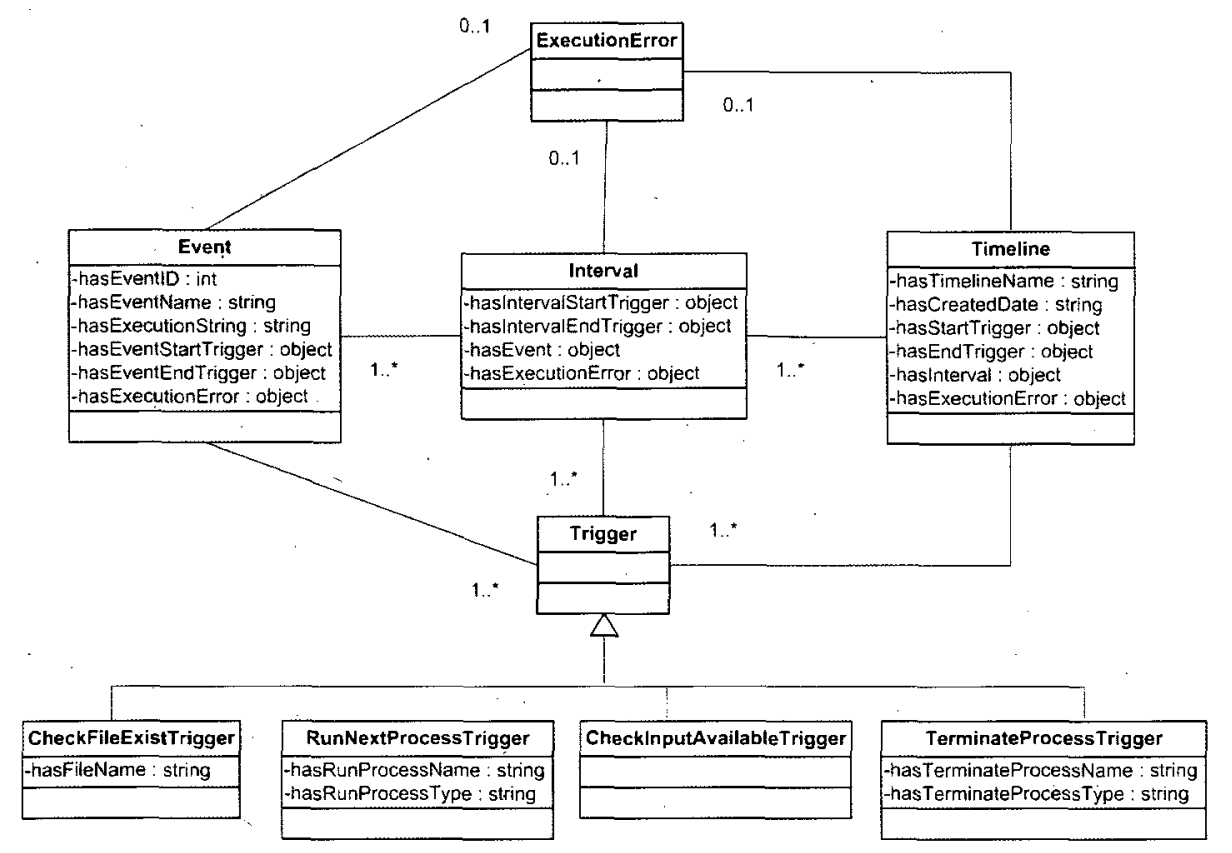

Figure 3.8: Properties of the Execution Timeline Ontology

defined in the Ontology. Even though not fully explored in this thesis error handling and other contingency management plans including early termination of the timeline and deadlock detection are attainable with use of this Ontology and possibly with the introduction of other Ontologies.

\subsection{The Access Class Libraries}

The system discussed in this thesis along with many other semantic web enabled applications are driven by a number of core Ontologies. Their functionality relies on the querying of ontological instances and their property values. This include the use of reasoning engines (in this case $\mathrm{FaCt}++$ ) to infer some knowledge which 
was perhaps never explicitly asserted. Two alternative approaches to achieving this knowledge acquisition from Ontologies exists. Generic OWL parsing APIs such as Jena and Protégé-OWL can be used to directly interact with the Ontology. These provide a model in which OWL instances and their properties are saved using generic Java classes [22]. As the application grows these may be rendered inconvenient since access to OWL objects is done through specifying the objects' names often as strings. This is rather hard to maintain as the Ontology evolves over time since every instance of a referenced (in the form of a string) but changed class instance or property value will have to be traced and modified. Another approach which is deemed to be the better of the two and adopted in this work is one in which in addition to the use of the models provided by the aforementioned APIs, access to the ontological facets is done through custom made Java classes that resemble the structure of the Ontology since the Ontologies are known to the application beforehand. These classes can be arbitrarily instantiated and extended by attaching more methods as deemed necessary by the developer of the application. These do not only allow seamless access to the Ontology but also provide cleaner object oriented design patterns. This class library consists of definitions of interfaces and classes that implements these interfaces. The interface is a normal Java interface that defines mandatory properties and methods that should be implemented by classes that commit to using this interface. The power of interfaces will be discussed in Section 3.3.2 where such issues as multiple inheritance will be examined. To illustrate this benefit consider the following example scenario that uses the Algorithm Ontology discussed in section 3.2 . 
The Algorithm and AlgorithmFile classes are defined in the Ontology. They define and hold instances of algorithms and actual algorithms files respectively. The relationship between this classes is through a hasAlgorithmFile object property whose domain is the Algorithms class and has AlgorithmFile as its range. The link is also provided via the inverse object property isAgorithmFileOf whose domain is the AlgorithmFile class while its range is the Algorithm class. The necessary Java class files that resembles this structure includes the creation of a class interface and a class implementation for each of these classes. This is demonstrated by Figures 3.9, 3.10, and 3.11 .

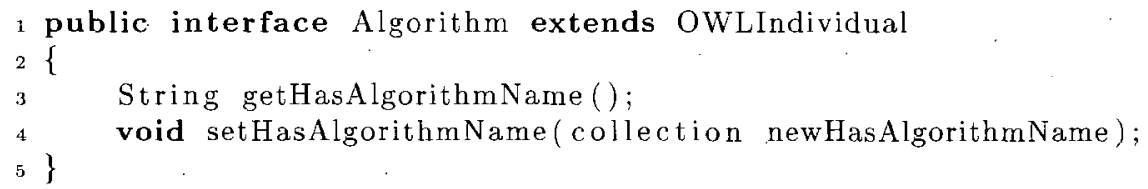

Figure 3.9: A Java example of the Algorithm Class Interface

There are other properties and methods that need to be defined for this class to be complete and fully functional, however, this will serve the purpose of putting things into perspective. With the definition provided in Figure 3.9 and 3.10 it becomes easier to reference and access the instances and their properties as you would other Java objects. Figure 3.11 demonstrates how this may be achieved. As is with any object oriented approach access to an object's properties and the methods that manipulate that object begins with instantiation of that particular object hence line 1. This is however done via a Factory class therefore myFac in this case is an object of that class. Once the object has been instantiated it becomes obvious 


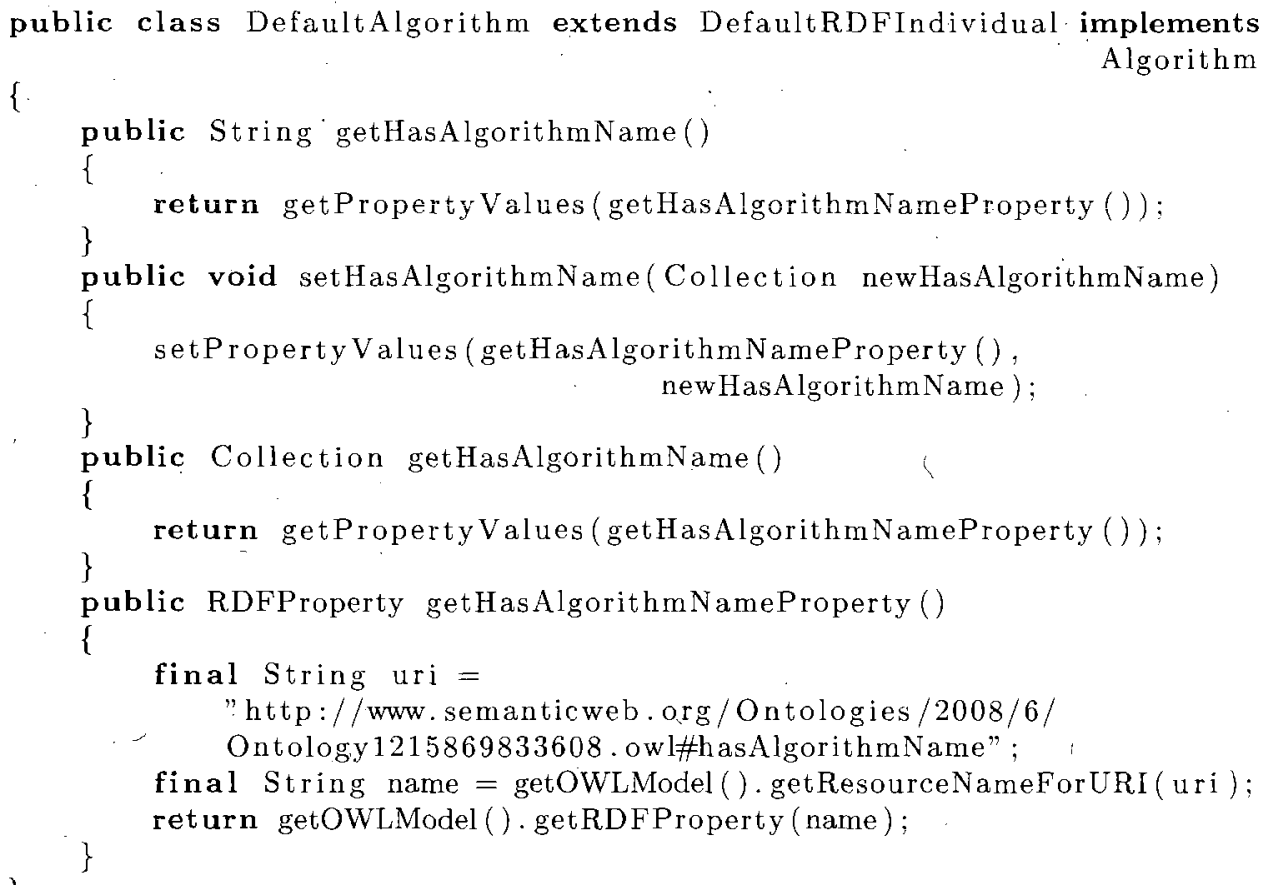

Figure 3.10: A Java example implementation of the Algorithm Class Interface then on how the methods are accessed (myAlgorithm.getHasAlgorithmName()) where myAlgorithm is the object and getHasAlgorithmName () is the method. What follows is an iteration on the results returned by the called method.

\subsubsection{Generation of Class Library}

The creation of custom Java classes that map to the Ontology classes is not a trivial process but one can manually create them. However, there exists code generators that can be used to ease this rather intricate process. This Java code generation capability does exist within the Protégé-OWL editor and it was used for 


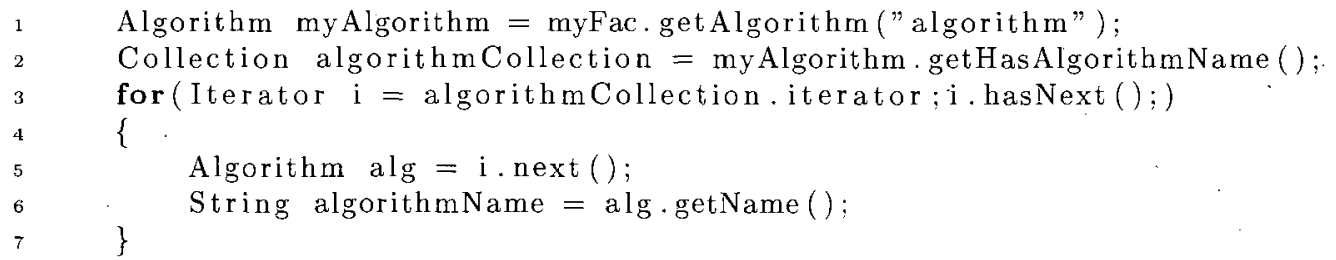

Figure 3.11: An example usage of the Algorithm Class

the generation of the Access Class Libraries used for the development of this system. Several other code generators exist; examples include OWL2Java and Jastor [43, 36]. The primary reason for the use of a Java class code generator was to delegate this task to mechanisms that would do it instantly and hence save considerable amounts of time. However, the code was then manual examined to identify omissions and physically amend faults. These code generators are designed to create a set of Java interfaces and implementation classes from OWL Ontologies such that an instance of a Java class represents an instance of an OWL class within the Ontology. As will be discussed in the section that follows the code generators may not represent all the classes and constraints within the Ontology solely because of the difference between Java and OWL. Manual inspection of the generated code and writing of the omitted concept may still have to be performed.

The use of code generators may be useful at the beginning of the creation of this framework however, this may fade. This is because at the creation of the Ontology many classes are created that are perceived to be needed, therefore manually creating a Java interface and an implementation class for each of the classes in the Ontology could seem to be cumbersome for a human being to do. The process would however, 
be quick and easy with the aide of code generators. The same cannot be said though after the framework has been set up and only small changes in the class structure are made. Changes could for example be the inclusion of a new class to accommodate a subclass that was overlooked. In this case the class can be manually created while leaving the others intact. The option to use the code generator is however, still open.

\subsubsection{OWL to Java Mismatches and their Fixes}

As mentioned earlier, it would have been ideal to have a one-to-one mapping of the Java classes to the OWL Ontology classes including their properties and asserted restrictions. However due to significant differences inherent within these languages this may not be attainable and therefore alternative ways need to be devised to minimize the impact of these differences. One of the major omissions by the code generator in our case was a someValuesFrom restriction placed on several data and object properties. The purpose of this restriction was to constraint the creation of property values to certain pre-listed classes and class instances. This implies enumerated classes. Enumerated classes are ignored by code generators since they are anonymous classes. This has little impact on our system since our primary focus is not on the creation of Ontology instances but rather their use to drive the development of a compositional system and in the cases where the population of the Ontology is done through an Ontology editor like Protégé this limitation would not be applicable. It would however be quite important for an application that alters the Ontology instances to handle this variation.

We do however recognize solutions and mappings provided in [20]. The 
paper suggests two rather simple solutions. The first solution involves the definition of an enum, struct or a list object that contains possible values for the property. The method that creates the property value will then loop through the enum checking to see if the new value is one of the possible values contained in the enum. The second solution is somewhat similar to the first one but it instead makes use of listeners registered on the restricted property which are invoked every time the property is changed.

Java is a single inheritance object oriented language while OWL is a very rich and highly expressive description logic based language that supports multiple inheritance. Java does not support what may be referred to as multiple implementation inheritance. It however allows a class to implement multiple interfaces. This is a feature that can be exploited to model classes that have two or more super-classes. We have however, managed to get away with not using multiple inheritance in the implementation of our Ontologies so this remains a feature that can be explored should the Ontology evolve and have classes that inherit from several other classes.

\subsection{Application Logic}

\subsubsection{Overview of the System's Functionality}

The idea behind this thesis is to have the users dictate which algorithms they want in a system and how these algorithms relate to each other. To demonstrate this possibility we have defined Ontologies and class libraries that allows for different architectures and ways in which algorithms can be orchestrated into a single 


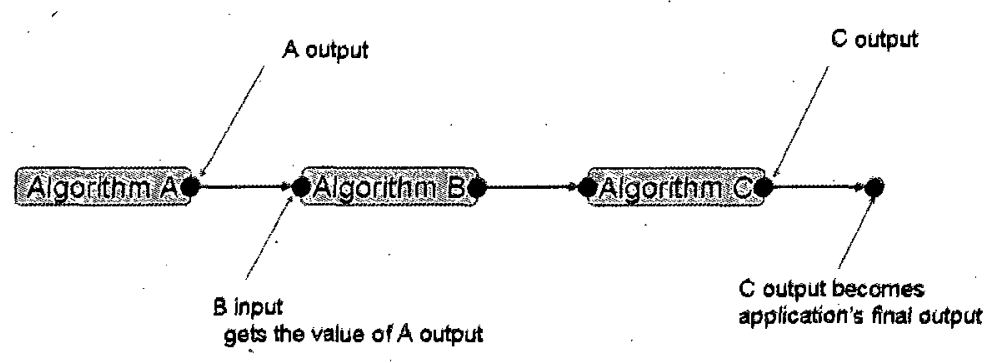

Figure 3.12: Overview of a Pipe and Filters style

unit that performs a needed function. Some of these include: a Pipe and Filters, a Vote, and simple concurrent running of several algorithms either synchronously or asynchronously. Please refer to chapter 4 section 4.1 .2 for definitions and details of these concepts. Figure 3.12 depicts an example of a pipe and filters design pattern. This architectural style is a data flow architecture that concerns itself primarily with the movement of data between data processing elements.

A vote can be seen as an arrangement where algorithms arè selected and run to each produce output that relates to a certain subject matter and their output is further analyzed to make a decision on that subject. This is analogous to the traditional ballot voting that we are accustomed to where participants place votes for a particular individual and a decision is made after counting the votes to determine the winner. A practical example of the applicability of this kind of arrangement is a syndromic surveillance system. This could be a system that has several algorithms operating on the same data set to detect outbreaks. However, these algorithms may not have the same sensitivity levels and may also have different configurations that impact on their alerting capabilities. Therefore one algorithm may signal the occur- 


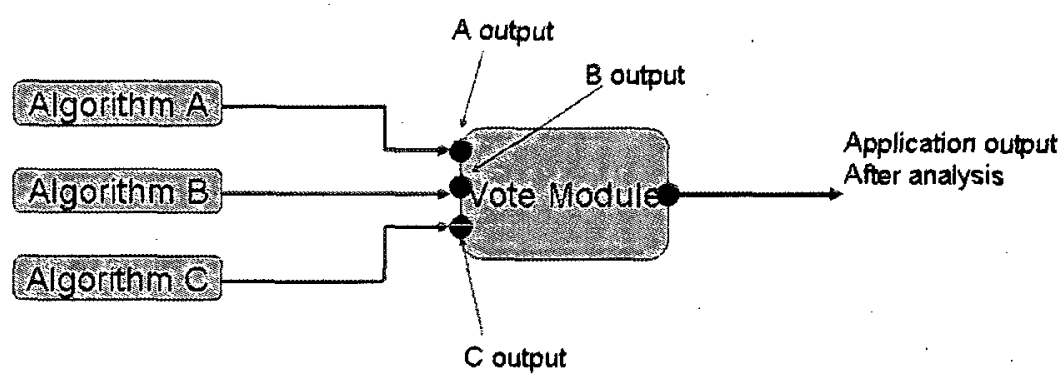

Figure 3.13: Overview of a vote design

rence of an outbreak while the other does not. It may then be practical to consider all participating algorithms' outputs before declaring or alerting the occurrence of an outbreak. Figure 3.13 depicts this arrangement.

The pipe and filters scenario presented earlier describes a situation where the algorithms are compatible to each other however, there may be situations where the output of one algorithm may not be compatible with input of the next algorithm and this incompatibility being the results of a difference in data types. Therefore rather than declaring the connection to be impossible a data type converter can be employed to rectify this incompatibility. All this decisions are aided by the Ontologies that define the algorithms, converters and other architectural elements discussed earlier. See Figure 3.14 for a graphical view of this pipeline with a bridge between the filters to enable connection.

The same kind of bridging does exist in a vote design; however, in a voting design this may be imposed by the voting pattern itself. A user may for example select Average as the voting method. This means that all algorithms run, produce their output and an average of these output is calculated from which decisions can be 


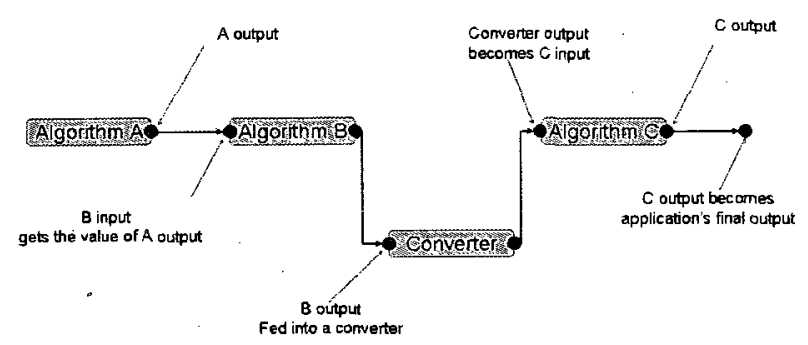

Figure 3.14: Overview of a pipe and filters style with another element providing a bridging function

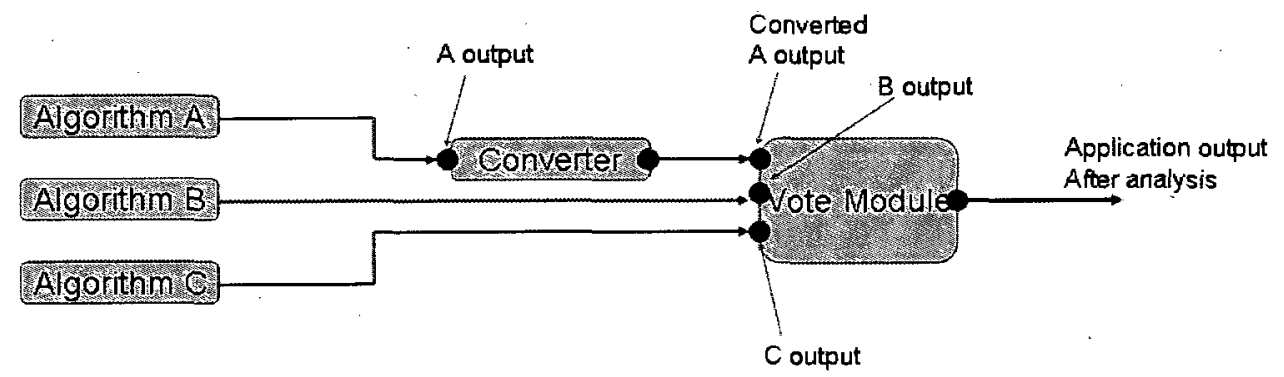

Figure 3.15: Overview of a bridged vote design

made. This implies that all output should be numeric therefore an attempt should be made to convert the non-numeric data type to numeric data types. Another example would be in the case where a Majority type of vote is chosen. This also implies that all participating algorithms' outputs should be the same data type for a realistic comparison therefore an attempt will be made to convert the non-matching data types to the majority data type before a decision can be made.

It should be noted though that incompatibility does not only result from difference in data types. Incompatibility could also be the result of the difference of data streams. One algorithm's output could be directed to the output stream while the algorithm it is supposed to connect to expects input from a file. Therefore routing 
the first algorithm's output to the second algorithm's input will not be possible. Our approach handles this type of mismatch by employing a module dedicated to this type of difference. This module is defined in the Ontology as an algorithm.

\subsubsection{Application Logic Framework}

In section 3.1 we presented a system architecture of which one of the components is the application logic. The application logic is the aspect of the architecture that controls the system's functionality. In this section we will provide a discussion of a framework for the application logic.

\section{Data Flow}

Since our idea revolves around selecting already existing algorithms and connecting them into a functional system, we have developed a framework to facilitate connectivity and the flow of data between algorithms. This was achieved by developing a collection of classes that defines a TaskGraph; Task, Nodes and Connectors. A Task is a representation of a unit that performs some function. For the purpose of this prototype system this can be in the form of an algorithm, a converter or any. of the architectural elements. A Task is created when the user chooses an algorithm or when an architectural element is invoked to provide for compatibility of the algorithms. A TaskGraph on the other hand is a collection of connected tasks. Nodes represent the inputs and outputs to an algorithm, converter or an element. They can take the form of file input and output nodes, standard input or output stream nodes, socket nodes and parameter nodes. We also define a Connector class that describes 
methods needed to establish a connection between algorithms. The possibility or absence of a connection being established between selected algorithms depends upon the compatibility of these output and input nodes. The connectivity also depends upon the availability of a glue component (either a converter or element) that will be placed in between the algorithms in case the algorithms' nodes are incompatible.

\section{Execution Timeline}

Once a TaskGraph has been established a walk through the TaskGraph will be done to extract and create events thereby creating an execution Timeline. This creation and ordering of events is dependent on the type of output and input of the sending and receiving algorithms. To illustrate this consider Figure 3.16. The Figure depicts a TaskGraph that has three connected Tasks. Task A pipes its output into Task B (this is possible since A writes to the output stream and B reads from the input stream). Task $B$ then writes its output to a file. Algorithm $C$ then reads the file and produces output of its own. This implies two Events: first Task A and B are run concurrently piping A's output to B and the output of B is written to a file; secondly Task C runs, reads the file produced by B and produces output. In this example scenario, the Timeline definition would have one Interval. The Interval would have one start Trigger that defines the next process to be run (Event 1) and the two Events. Event 1 would have an end Trigger that specifies Event 2 as the next process to run. Event 2 on the other hand would have a start Trigger that checks for the existence of the input file before it could start otherwise the Event will terminate with an appropriate error and ultimately the Timeline will terminate 


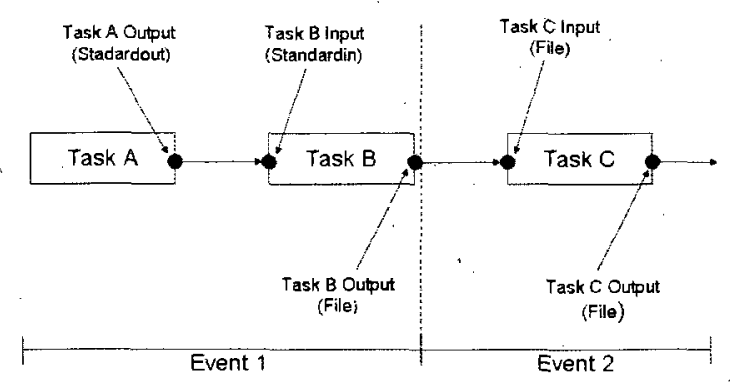

Figure 3.16: An example TaskGraph and the extraction of Events.

too. The Interval will also have an end Trigger that signals that the Interval is the last within the given Timeline and therefore providing an end point for the Timeline. Figure 3.16 is yet another example of a pipes and filters architecture. This architectural pattern is trivial to construct from the ODSC Ontologies and can itself be stored in an Ontology that describes architectural design patterns.

\subsection{Composing a System and Ontology Manipulation}

The process of composing a system involves three major steps; first the population of the algorithm Ontology with instances, secondly the system is composed by selection of desired algorithms and arranging/connecting them in the desired order, and lastly running the application. This section discusses what we envision as the ideal method of composing a system based on the Ontologies described in this thesis and the framework provided. We then follow this with discussion of how composition and Ontology manipulation was conducted in demonstrating the aspects of this prototype. 


\subsubsection{Ideal Method of System Construction}

Since we are advocating for reducing the amount of effort required of the users it is ideal to have a Graphical User Interface that has features that would help ease the construction of an application. Ideally the domain expert should be able to have at his disposal a list of algorithms (maybe the results of a search), a list of each algorithm's inputs and outputs, the ability to connect and disconnect any number of algorithms. This is all possible since the back bone is already setup in the form of packages and classes, all that is required then is to invoke the appropriate methods of the relevant classes. In addition to the domain experts, contributors of algorithms should have the means of publishing and describing the aspects of their algorithms.

What is deemed to be the right user interface can be a very subjective matter. This is because domains differ in functions and needs and therefore the design of a user interface needs to be specialized in that regard.

\subsubsection{Current Setup}

For the purpose of this demonstration the instances of the algorithm Ontology were created and manipulated through the use of the Protégé Ontology Editor. A test class was used to programmatically invoke relevant methods for composition of a system the same way they would be through a user interface. For the sake of brevity while maintaining clarity let us consider the following trivial example scenario: Suppose we have three programs; dummyFileWrite that writes a series of numbers to a file, genBool that reads data from the standard input stream and prints to the screen 
either a true or false based on some condition (eg. if value greater than five then print true otherwise print false) and lastly File ToStdout that reads values from a file and prints them to the output stream. Suppose the user wants to determine whether to associate either a true or a false to each of the values in the file. The user can make use of dummyFileWrite and connect it to genBool to achieve this. Figure 3.17 shows how this would be achieved in the test class.

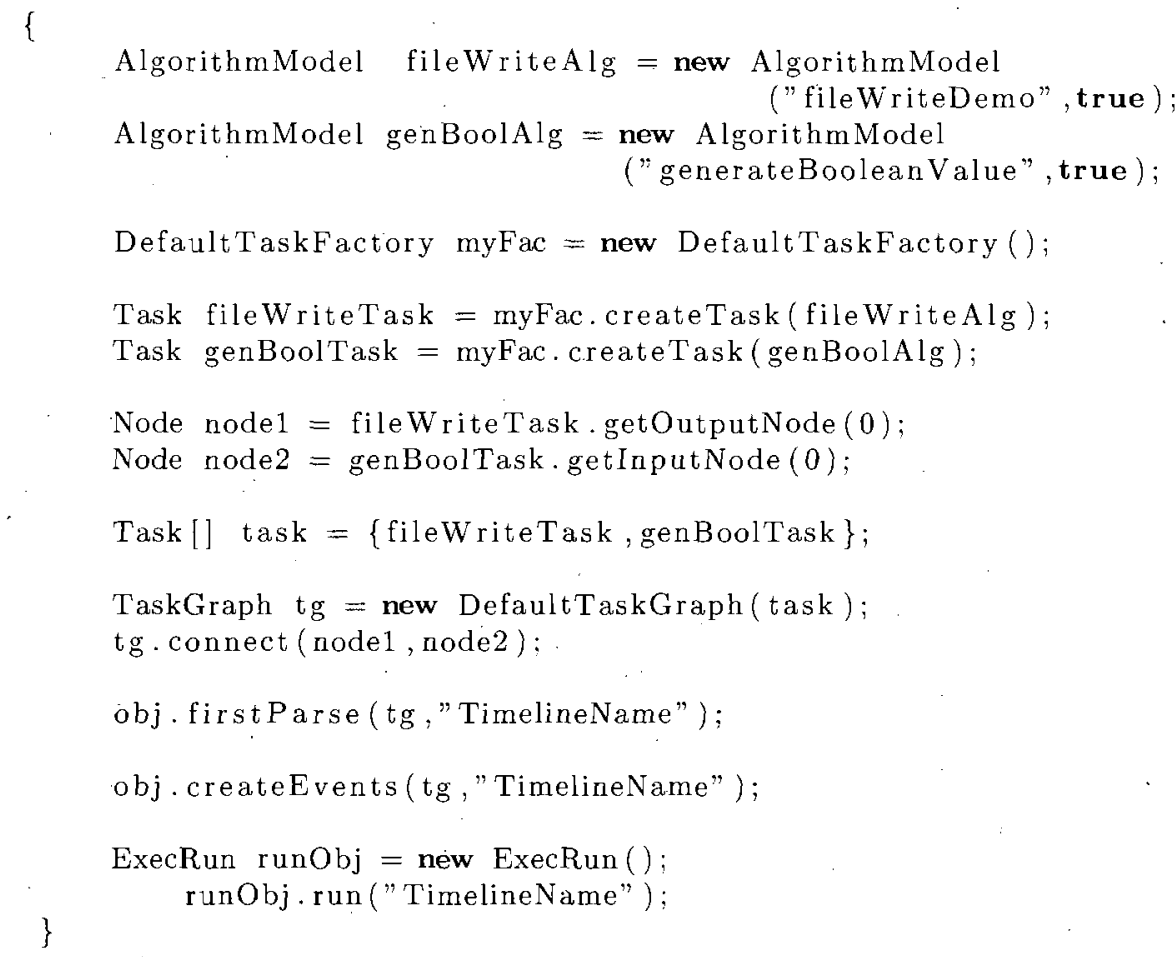

Figure 3.17: Example Test Class: Creation of Tasks

The first three lines of the code (line 1 to 3 ) create objects of the desired algorithms with an option to retrieve the properties of the algorithms from the Ontology (note the boolean parameter passed to the AlgorithmModel constructor). The 
next thing then (line 7 ) is to create a TaskFactory object that we use to create our tasks (lines 9 and 10) (note that a task is basically a representation of an algorithm). We then retrieve the output node of the sending (fileWriteTask) task (this can be achieved either by specifying the node index or the name of the node) and the input node of the receiving (genBoolTask) task (lines 12 and 13), after which we create a task graph that contains the two tasks we previously created (line 17). Then we connect the output node of the sending task to the input node of the receiving task (line 18). Remember though that the nodes being connected are not compatible as one is a file output node while the other one is a standard input node expecting input through the standard input stream. This is automatically handled by the connect method in the task graph by creating another task and placing it in between the two tasks. This task is basically another program (FileToStdout) that compensates for the difference of the streams. Lines 20 and 22) are responsible for the creation of the order of execution (Timeline) by first running through the task graph to determine where the events will be and finally creating the events (obj is an instance of the setupTimeline class responsible for the creation of timelines and events). This would complete the task of creating a timeline;however the created timeline will have to be executed and hence line 24 and 25 . The runObj object in line 24 is an object of a class responsible for traversing through the timeline and executing the events according to their respective order. The example task graph depicted by Figure 3.16 resembles a task graph of this scenario. All these details could be encapsulated through the use of a graphical user interface. 


\subsection{Assumptions}

We assume that the Ontologies have already been populated with instances or at the bare minimum that there is a way of loading the knowledge into the Ontology." We assume this because even though this is not a trivial process it has not been implemented in this thesis. Two alternatives exist though for loading the data into the Ontology; using the Protégé-OWL tool or to create an interface that can interact directly with the Ontology file to add and modify the class and property individuals. For the purpose of this demonstration we have used the former to populate the Ontology with instances since we have not yet developed an user interface. The Protégé editor has a Java graphical user interface for manipulation of instances. Restrictions such as the "valuesFrom" are handled by only availing to the user values that are allowed for a particular property in the form of a list that the user can select from. In the future the user interface to be adopted for the system will need to emulate this functionality.

Having made this assumption enables the development of this composition system to carry on without concerns about certain OWL to Java mappings that could not be realized during the generation of Java classes from the Ontology classes using the Protégé-tool such as enumerated classes within the Ontology. Refer to section 3.3 for details on mismatches in Java classes and OWL classes.

It is also assumed that the algorithms to be run exist as executables files or compiled Java class files in the case where they were written in the Java programming language. It must be noted though that the algorithm Ontology has almost 
all the information needed to compile the files in the cases where they only exist as source code files or at the least they are extensible enough for this information to be incorporated. In addition to this, there will be a need to code the logic which uses this information to do the actual compilation of the files.

\subsection{Summary}

The idea behind this thesis is to have the users dictate which algorithms they want in a system and how these algorithms relate to each other. To demonstrate this possibility we have defined Ontologies and class libraries that allows for different architectures and ways in which algorithms can be orchestrated to form a system. This demonstration was through the design and development of a prototype system using the Java programming language driven by two main Ontologies; an algorithms Ontology and an execution timeline Ontology. This was done by building on current research in the use of semantic webs for application development by mainly adapting a. system architecture that details the components of the system and how they all fit together.

A semantic web application is characterized by two layers; first, there is a semantic web layer that hard-codes knowledge about a particular domain in the form of Ontologies which is then used to model the behavior of the application. Secondly there is an internal layer which is composed of the reasoning mechanisms and the application's control logic that controls the application's functionality and interacts with the user via an interface. The prototype system takes advantage of 
this architectural structure.

The use of Ontologies to provide domain specific knowledge base in order to facilitate communication and knowledge sharing between people and various computer agents is arguably the pillar on which the semantic web hinges. We use the common knowledge provided by these Ontologies to drive the development of a compositional system by using the Ontologies to provide an extensible description of the components of a system and how they can be put together. The algorithm Ontology for the prototype system discussed in the thesis provides an extensible standard description of algorithms in the context of the elements or aspects of the algorithm that one would need to know about the algorithm in order to use the algorithm. The execution timeline Ontology describes the order in which the connected algorithms that form a system will be executed.

Class libraries that form part of the internal layer are used to access and manipulate the ontological instances. These are Java classes that map to the class structure of the Ontology. These classes are constantly instantiated and used in the application logic that controls the overall functionality of the system. This application logic also defines other sets of libraries that form the base of the system such as the data flow library that defines the framework for controlling the flow of data within a composed system. 


\section{Chapter 4}

\section{Case Studies}

In this chapter we provide a description of the case studies used in this thesis. We. start off by providing a context for the case studies by giving definitions of concepts that relate to the case studies and a brief discussion of the purpose of the case studies. We then provide the details of the case studies. A detailed analysis of the case studies is provided in chapter 5 (Analysis).

\subsection{Background and Purpose}

This thesis set out to research the aspect of system composition where a system is designed by simply making use of already existing algorithms and ordering them to fit a specific purpose. The guiding principles for the research had been ease of creation and extension of a system in as far as inclusion of algorithms, exclusion of algorithms, manipulation or changing the parameters that affect the overall functionality of the algorithms and support or use of certain architectures that deals with the data flow between algorithms that may ultimately affect the systems output. As a result of the research an Ontology driven prototype system was proposed and developed. 
The case studies, therefore, serves the purpose of illustrating the aforementioned characteristics of a semantic web composition system. The chosen case studies intend to illustrate three general aspects of the system under which these "characteristics fall: (1) The composition aspect of the system, (2) The architectures and the possibility of extension, and (3) Software Quality Attributes.

\subsubsection{System Composition}

The fundamental elements of a system are the components that perform computation; in our case these are the individual algorithms. Composition therefore concerns itself with bringing together these algorithms to perform a given function. Different types of algorithm elements are discussed in this thesis and the chosen case studies illustrate how these elements are harmonized in a systematic manner to produce a desired output. These algorithms include (1) Architectural elements that are custom made and included in the system to provide a "gluing" functionality in as far as connecting algorithms, data flow, data type conversion, file manipulation etc. (2) Computational elements which do the actual functional computation. These range from custom made to already packaged algorithms that may be domain specific or generic in purpose.

\subsubsection{The Architectures and the Possibility of Extension}

In as much as the output of the system is dependant upon the algorithms that work on a given data set and the data itself, several architectures and architectural elements also have a direct impact on the outcome. This is because they may 
affect the ordering of the algorithms which in turn has direct implications on the flow of data between the algorithms. In addition to the order of the algorithms the data flow is also affected by the compatibility of the connected algorithms' outputs and inputs in which case the architectural algorithms discussed earlier may be employed to complete the connection. In as far as data flow is concerned several architectural concepts are relevant; these include pipes and filters and the forever popular file writing and reading.

Pipes and Filters A pipes and filters architectural style is a dataflow architecture that concerns itself primarily with the movement of data between data processing elements [37]. To better define a pipes and filters architecture we first define the components that comprise pipes and filters. Filters are defined by Taylor et al as preexisting components/programs that consume data from the input stream and produce data through the output stream [37]. Their basic function is to perform arbitrary processing of data that may include transformation and enrichment of the data. Pipes were defined by the same authors as connectors that interconnect two filters by offering buffering functionality and routing of the first filter's output stream to the input stream of the second filter. It goes without saying then that a pipes and filters architecture is an architectural style that defines several executable programs(filters) that are executed possibly concurrently and employing pipes to route data streams between the programs. This type of architecture allows for the creation of applications even by people without prior software development training. 
Concurrency The architecture discussed above also brings into mind another interesting architectural concept: concurrency. To provide a context for understanding it let us preface the definition of concurrency by contrasting the platforms(granted there is ambiguity in the use of this term) that systems run on. In the past and God forbid even today systems were designed to run on a single computer that has a single processor, contrast that with the existence of multiprocessor computers where simultaneous running of multiple task can be done [37]. Furthermore the existence of networks of computers that in turn has either single or multicore processors presents another platform. Therefore concurrency presents itself in multiple tasks being performed simultaneously either by employing multiple threads or system processes in the case of a single host and different hosts in a network running independent processes that cooperates to complete a system function. Even though most architectural styles have the notion of concurrency, it can also come as a deliberate decision in which several algorithms are selected and all run simultaneously with each producing output.

Combinations Other interesting architectural concepts explored in this thesis and hence in the case studies are Combinations. These are arrangements where multiple inputs are fed into a component which in turn produces output by manipulating the inputs. The inputs to the component could for example be output of several algorithms that is fed into a component for processing. Examples of combinations include but are not limited to: Average-taking an average of the inputs, Min-the minimum value from the inputs, Max-the maximum value from the inputs, Vote- 
making a decision on a subject matter based on how many of the inputs are for a given subject matter and how many are not in support of the subject matter.

In this case study we will only illustrate one of the combinations, a vote. A vote manifests as a conscious decision by the user to have several algorithms run each producing output which is then fed into another module that takes a decision (vote) on the subject matter and produce some output. Vote types may differ examples may include:(1) Majority vote- a decision made based on the principle that for a positive decision more than half of the votes cast are for a particular subject and vise versa, in our case more than half of the candidate algorithms indicates an alert. (2) Weighted -Vote-in this type of vote the vote cast by other candidate algorithms is given more weight than the other algorithms in determining the overall outcome. We however only illustrate a majority vote in our case studies.

The chosen case studies' overall purpose is therefore to illustrate the use of the architectures discussed earlier and how easily extensible the system is in as far as introduction of other architectures, modification of the present and inclusion of more algorithms into a system. All of these are facilitated by the use of Ontologies to provide descriptions of the components and the order of events execution.

\subsubsection{Software Quality Attributes: Flexibility, Reusability, and Testabil- ity}

In as far as software quality is concerned our primary objective is to illustrate how several aspects of the prototype system relate to and address the aforementioned 
quality factors. To establish a context it is fitting therefore to first provide some background and definition of software quality. We then narrow the definition down to definition of each of the aforesaid quality factors.

A large collection of literature has emerged over many decades on quality and software quality specifically. However there appears to be no universally agreed upon definition of the phrase "Software Quality" [4,30]. In fact Cavano et al [4] flags this lack of agreement on the definition of quality as a source of confusion in as far as defining the quality goals for software. Some of the definitions of software quality include among others the view of software quality as conformance to specifications $[19$, 30]. Without disqualifying this definition an obvious doubt exists as to whether this definition holds when the software followed flawed specifications. Another definition provided by. Plant [30] relates software quality to the extent to which the software is perceived or judged by the user to exceed their needs and expectations.

In the quest to establishing better quality assurance methods a trend in the literature has become evident. This trend revolves around defining software quality in terms of the characteristics (Factors) of the software by defining quantitative measures(Metrics) of these attributes and coming up with conclusions of the level at which the software has that particular attribute. Therefore further definitions of software quality exists such as one by the IEEE Standard 1061-1998 [18] where software quality is defined as "the degree to which software posses a desired combination of attributes". This implies an explicit listing of the desired attributes and articulation of specific measures for these attributes in determining the quality of or absence of 
software quality.

A model for the measurement of software quality proposed by McCall [4] presents a triad of perspectives for defining the quality of a product: Product Revision, Product Operation, and Product transition. The model further details these perspectives into a hierarchy of factors, criteria and metrics. This framework is depicted by Figure 4.1. For the definitions of the quality factors of interest to us we follow the definitions provided in this article. Flexibility was defined as the effort needed to change a functional program. Reusability refers to the extent to which a program can be used in other programs. While testability refers to the ease of validating a program to ensure it does its intended function.

Boehm et al. [2] also proposed a model whose objective was to define software characteristics and various metrics to asses the degree to which the software has the said attribute. This model differs from McCall's in that McCall focuses primarily on measurement of the high level characteristics while Boehm's model is based on a wider range of characteristics. The similarity though is that for both models a set of metrics are defined to measure the specified attribute.

The aforesaid models were used as the bases for the ISO 9126 standard as is evident in the similar structure of this standard. This standard specifies six quality factors for software evaluation in functionality, reliability, usability, efficiency, maintainability and portability [28]. 


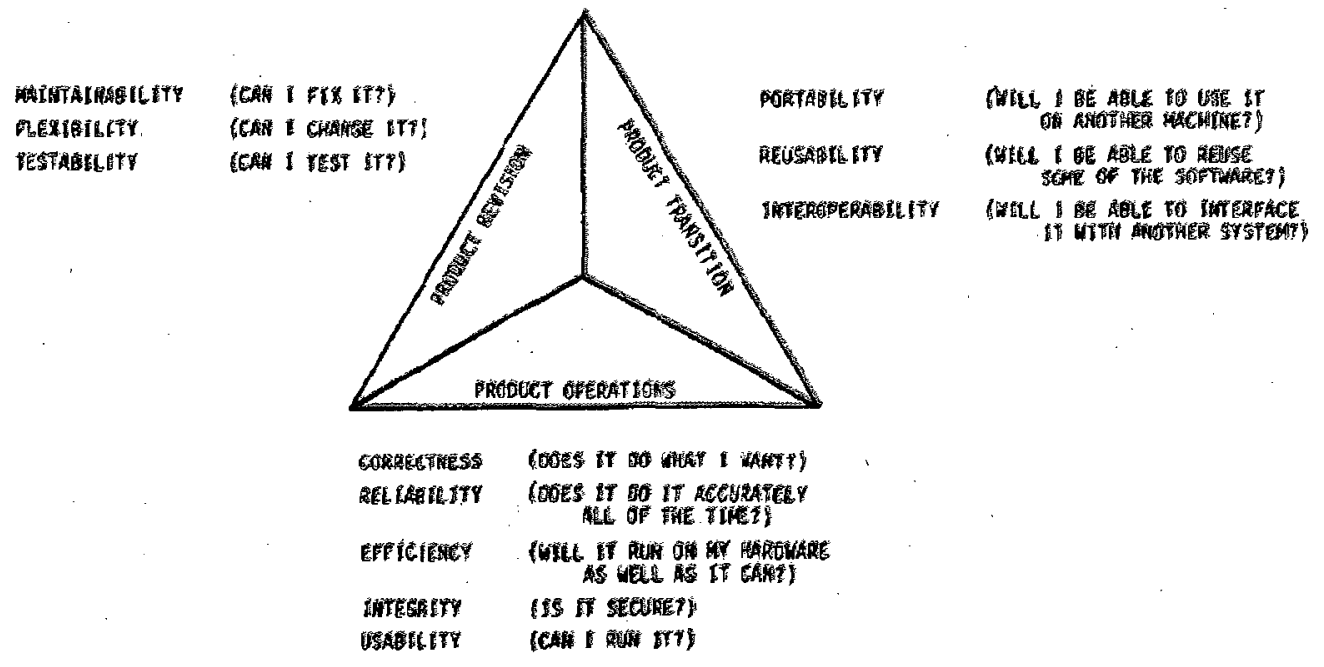

Figure 4.1: Overview of McCall's Software Quality Factors (source:[4])

\subsection{The Case Studies}

Syndromic Surveillance was the initial motivation of this thesis. This is because it operates in a very dynamic environment and hence requires rapid system development, modification and the deployment of various detection algorithms for a timely detection of disease outbreaks. Please refer to section 1.1 for details on the motivation of this thesis. The case studies are therefore chosen to mimic some of the functionality of several popular syndromic surveillance systems. This is done by employing some of the algorithms used in those systems as discussed in section 4.2.1. These are purelý statistical algorithms that work on time series data to offer early signals on the occurrence of outbreaks. It should be noted, however, that Syndromic Surveillance is used as an example application area. The Ontologies used in this thesis are very generic and extensible állowing for this system to be adapted or used 
in any discipline.

\subsubsection{The Algorithms}

As discussed in section 3.3 the structure of the classes in the system is relatively similar to that of the class structure of the algorithms Ontology. Therefore each algorithm object is easily populated with instance defined in the Ontology. Figure 4.2 depicts this class hierarchy making it relatively easy to identify where each of the algorithms discussed below fall.

Early Aberration Reporting System (EARS). The quality control cumulative sum methods from EARS are used in the case studies. These includes the variants of the CUSUM method: C1-Mild, C2-Medium, and C3-Ultra. Refer to [17] for details of these implementations.

Real-time Outbreak and Disease Surveillance System (RODS). From RODS the algorithm used in the case studies is the Recursive Least Square (RLS) algorithm. Refer to [39] for a detailed description of the algorithm.

Electronic Surveillance System for the Early Notification of CommunityBased Epidemics (ESSENCE). We also make use of the Exponential Weighted Moving Average (EWMA) algorithm used by ESSENCE to give epidemiologist clues on the occurrence of a disease outbreak. Refer to [3] for the details on ESSENCE and the algorithms it employs. 


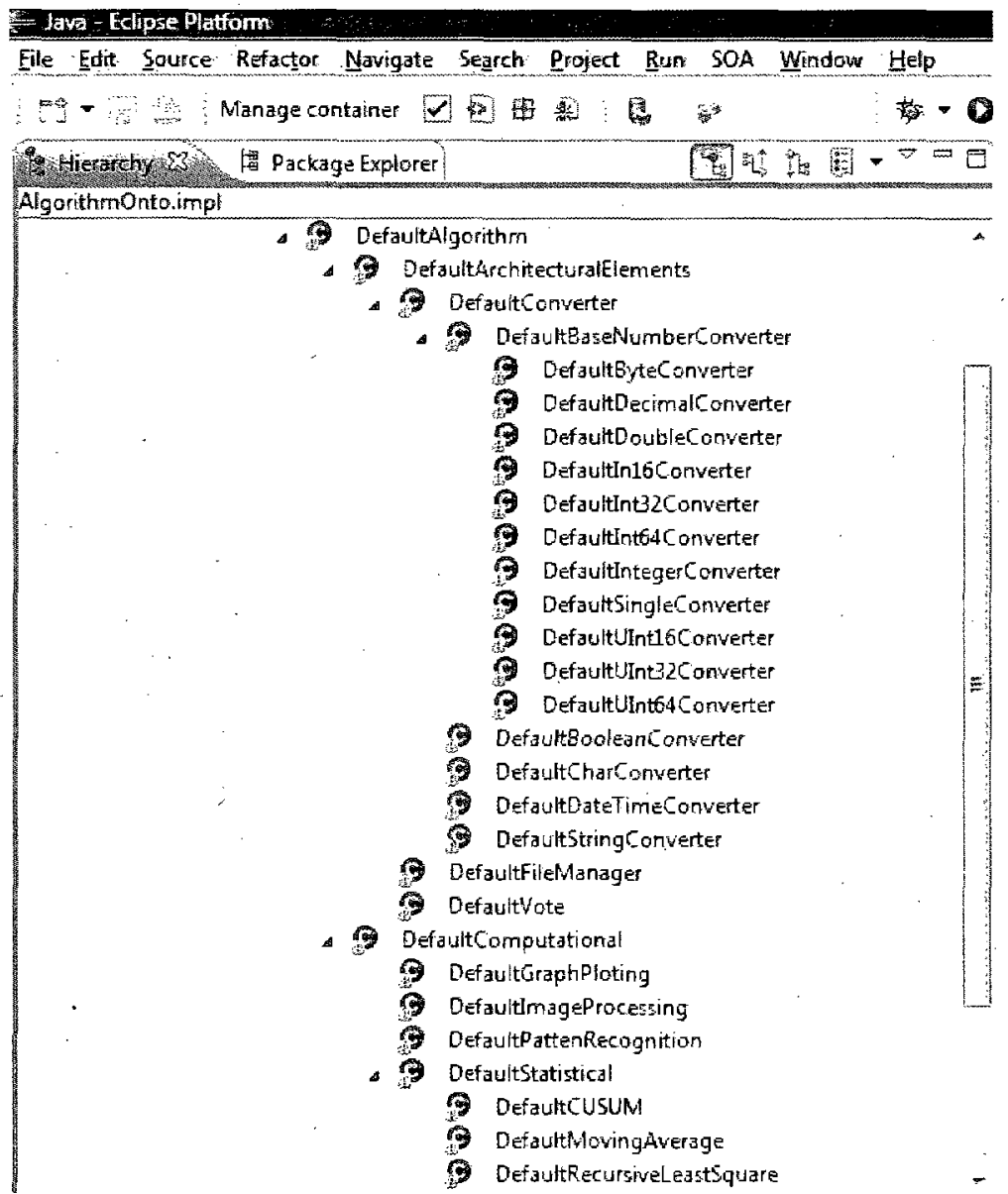

Figure 4.2: Algorithm class hierarchy(Java Classes) 
Packaged Algorithms. In some of the case studies we used an open source graphing tool called LiveGraph. LiveGraph is a customizable data visualization tool that reads data from a text file and plots it into a graph.

Custom-made Algorithms. These include a majority voting module, FileMergeran algorithm that consolidate several files into one comma separated value file, several data type converters, a module that reads data from a file and prints to the output stream and a module that reads from the input stream and writes to file. The last two are needed to help in a connection where the input and output streams of connected algorithms differ.

The first step in setting up the case studies is the publishing or describing of the algorithms in the Ontology. This is done by defining ontological instances of the individual classes in the Algorithm Ontology that describes several important aspects of the algorithm in question. The Ontology is setup in the context of describing what an algorithm is and what would be needed to run an instance of that algorithm. Refer to section 3.2 for the details on the Ontology. An algorithm is defined in the Ontology relative to the other algorithms within a specific class that it belongs to. The description of an algorithm involves three steps: defining an instance of an algorithm within a specific class, defining the details of the environment needed to run the actual executable of the algorithm, and defining the inputs and outputs of an algorithm instance. As an example the Cumulative Sum C1-Mild algorithm mentioned earlier will be an instance of the CUSUM class. This is a class that defines general properties that apply to cumulative sum implementations. Should the need 
arise definition of subclasses of this class can be done to cater for more specific properties relevant to an implementation of a cumulative sum algorithm. Then descriptions of the actual algorithm file are defined in the AlgorithmFile class. These include definition of whether the file is an executable file or source code, the location of the file, the language it was written in, the run prefix (the command needed to run the file) etc. It should be noted though that the only file types that has been used thus far are executable files (.exe) and compiled Java files (.class) however this should not be construed to mean that our implementation is limited to these since it is extensible enough to have other file associations. Following our C1-Mild example the algorithm file used is an executable file written in the $\mathrm{C}$ programming language and has no run prefix since it is an executable. This information is therefore published in the relevant properties.

Last but not least the definition of the inputs and outputs of the algorithm are provided for in their respective classes. Each of these classes is further sub divided into subclasses to provide specific definitions of the inputs and the outputs. For example, a DataFiles subclass of the input class defines a C1-Mild input instance along with the relevant properties that may include among others the filename, the file delimiter, the column of interest etc. This is rather a summarized process followed in defining the instance of the algorithms to be selected as components of the system.

Having defined the algorithms in the Ontology provides a centralized place for altering the parameters of the algorithms and thereby altering the functionality. As an example modifying the window period property of the EWMA algorithm will 
have an impact on the averages calculated by the algorithm and hence the alerting sensitivity. A published or described algorithm is therefore available for inclusion into a system.

\subsubsection{The Data}

Since large scale and well documented outbreak data is pretty hard to come by it is reasonable to test systems using synthetic data. This is time series data that has known outbreaks injected in it. It then follows that the case studies in this thesis use synthetic data.

\subsubsection{Case Study 1: Concurrent Running of Algorithms}

In our first case study we selected four algorithms: RLS, C1-Mild, C2Medium, and C3-Ultra. These algorithms are domain specific statistical algorithms that do the actual work of outbreak detection based on provided time series data. As mentioned earlier all of these algorithms' details are published in the Ontology including all the relevant parameters they need to be run. Since these algorithms are independent of each other they were run concurrently (each without concern of whether the other has completed execution) to each other with each producing output. These algorithms write either a one or a zero to a file for each day to indicate an alert or otherwise respectively. The algorithms could however produce any type of output that could be passed to either other systems or programs to determine the presence or absence of an outbreak. These output files can then be further used in whatever way the epidemiologist wants; this may include feeding it into another program as 
input, plotting it into a graph for better visualization, analyzing it to get a measure of the accuracy of the algorithms etc.

The description given above is what the user perceives to be happening; what happens behind the scenes though is a whole other process. Whenever a user selects an algorithm for inclusion a Task is created and eventually a TaskGraph (a collection of Tasks) is created. This TaskGraph is then sent to another module that loops through the graph creating an order of events execution in the form of a Timeline. This creation is guided by the dependencies that exists between the different tasks of a graph. The dependencies are established when the tasks are connected at design time. However, in this case study the tasks are never connected and therefore seen by the module as four independent events that should be run concurrently and independent of each other.

What we end up with is a timeline defined in an Execution Timeline Ontol, ogy. Refer to section 3.2 for details on this Ontology. In this case study the timeline created is a simple one that has one Interval and four Events. In addition to the events the interval also has a set of start and end triggers. The function of the start trigger ranges from checking the preconditions needed to run the interval to indicating what the next process to run will be. The end trigger could indicate the end of an interval and could also serve the purpose of indicating what to do next. In this case study the start trigger served the purpose of indicating the four events as the things to run next. For each event created, event start and end triggers are also created. These triggers do the same thing as interval triggers. In this case study the start 


\begin{tabular}{|c|c|c|c|c|}
\hline Interval & Event Name & Dependency & Algorithm & Output \\
\hline \multirow{3}{*}{ Case1Interval0 } & Case1C3UEvent0 & - & C3-Ultra & c3results.txt \\
& Case1RLSEvent1 & - & RLS & rlsresults.txt \\
& Case1C1MEvent2 & - & C1-Mild & c1results.txt \\
& Case1C2MEvent3 & - & C2Medium & c2results.txt \\
\hline
\end{tabular}

Table 4.1: The created events in case study 1

trigger created for each of the events was a check file exist trigger (since input to these algorithms is a data file). This is a precondition that checks if the input files to these algorithms exist and only if this condition is met will the event run. Exit points are provided for within the timeline in the form of error handlers. An event definition includes along side definition of triggers a definition of an error handler. The same applies to intervals and also to the timeline. This provides a flexible platform where different types of error handlers can be built and incorporated. The system will exit at whatever point an error occurred.

Table 4.1 presents a summary of this case study. The first three columns relate to the various events of the timeline described in the Ontology, while the forth column specifies the actual algorithms that the events relate to, and the last column specifying the output assaciated with each event after the timeline is executed. A pictorial view of the timeline described for this case study is also depicted by Figure 4.3. Refer to table 4.1 to see the algorithms that corresponds to each of the events in the timeline figure. 


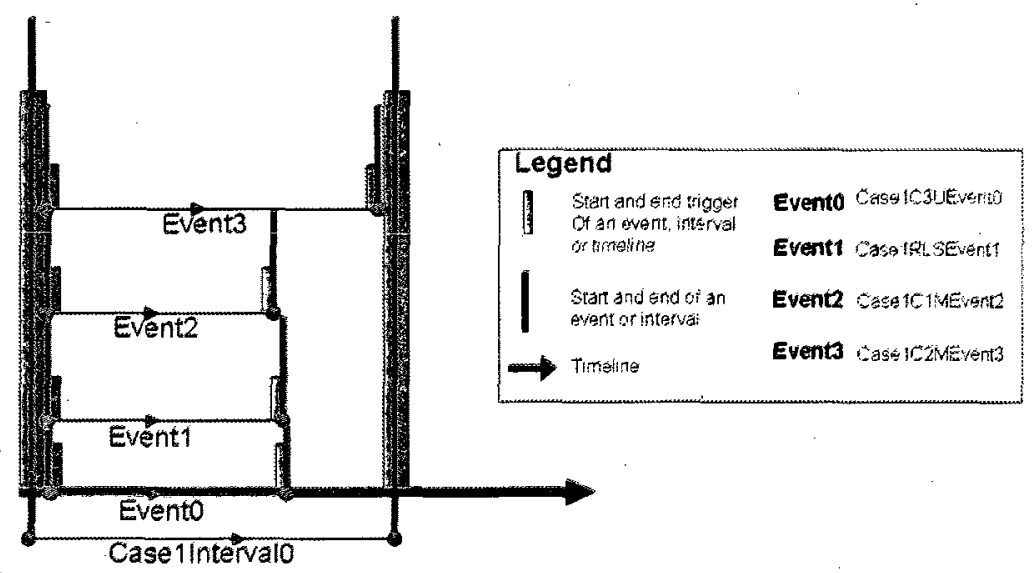

Figure 4.3: Overview of case study 1 timeline

\section{case study 1.1 Connection and Use of Algorithms' Results}

Here we discuss a variation of the case study discussed earlier. Instead of having four independent algorithms running at the same time we altered the arrangement to include two more algorithms: FileMerger and LiveGraph. FileMerger is a custom made module that aggregates algorithm outputs into one comma separated value (CSV) file, while LiveGraph is a packaged open source tool that plots data into a graph. In this arrangement we have each of the four algorithms now connected to the FileMerger algorithm and LiveGraph works as a separate event that uses the CSV file as input. This implies dependency of the FileMerger on the successful completion of the algorithms connected to it. The output file created by FileMerger can be seen as a collection or a buffer that can then be accessed by another program or system that understands its format. This was done to illustrate the extent to which this implementation is extensible and how useful it can be. The results and events of this case study are depicted by table 4.2, Figure 4.4, and Figure 4.5. Figure 4.5 shows a 

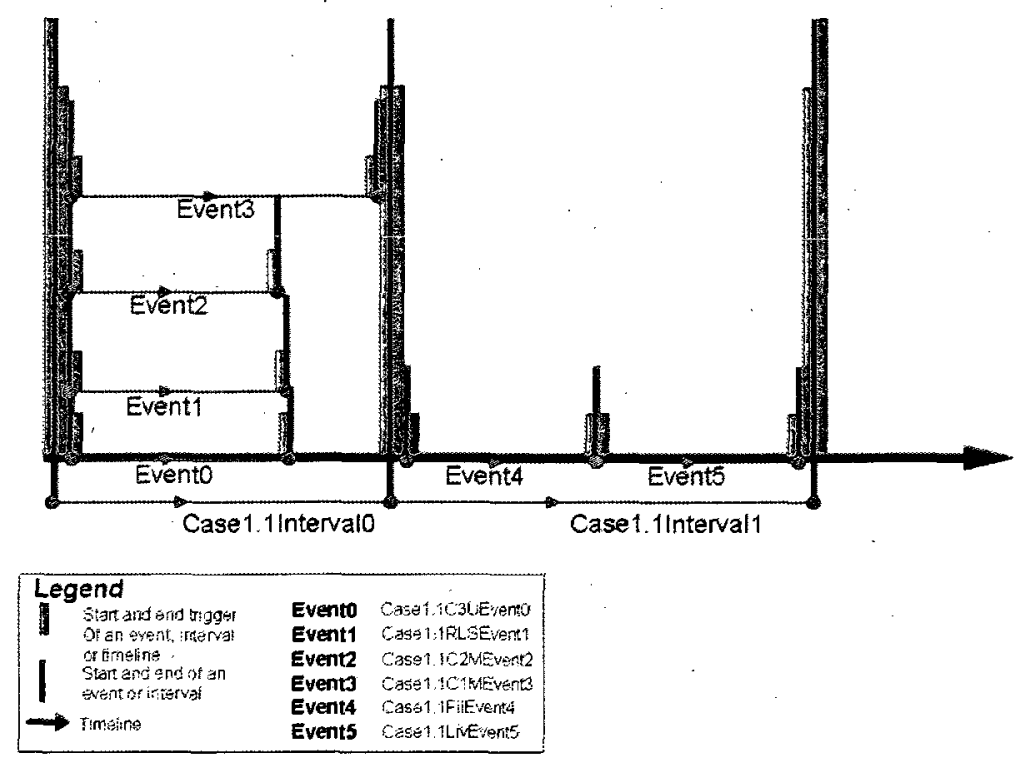

Figure 4.4: Overview of case study 1.1 timeline

LiveGraph plot of the case study 1.1 timeline. Some of the lines overlay each other because data points of the algorithms for that particular day are at the same place therefore some of the lines are not visible. Clicking on every data point on LiveGraph reveals which algorithms share that data point. That functionality is not available in print therefore to mitigate for this the figure was edited to show which algorithms alerted at each of the data points. Only one algorithm (C1) alerted on day 19, three algorithms (C2, C1 and $\mathrm{C} 3$ ) alerted on day 20, and only two (C2 and C3) alerted on day 21.

\subsubsection{Case Study 2: Voting}

This case study is somewhat similar to the above case study, however a small variation is made. This case study makes use of the three CUSUM variants 


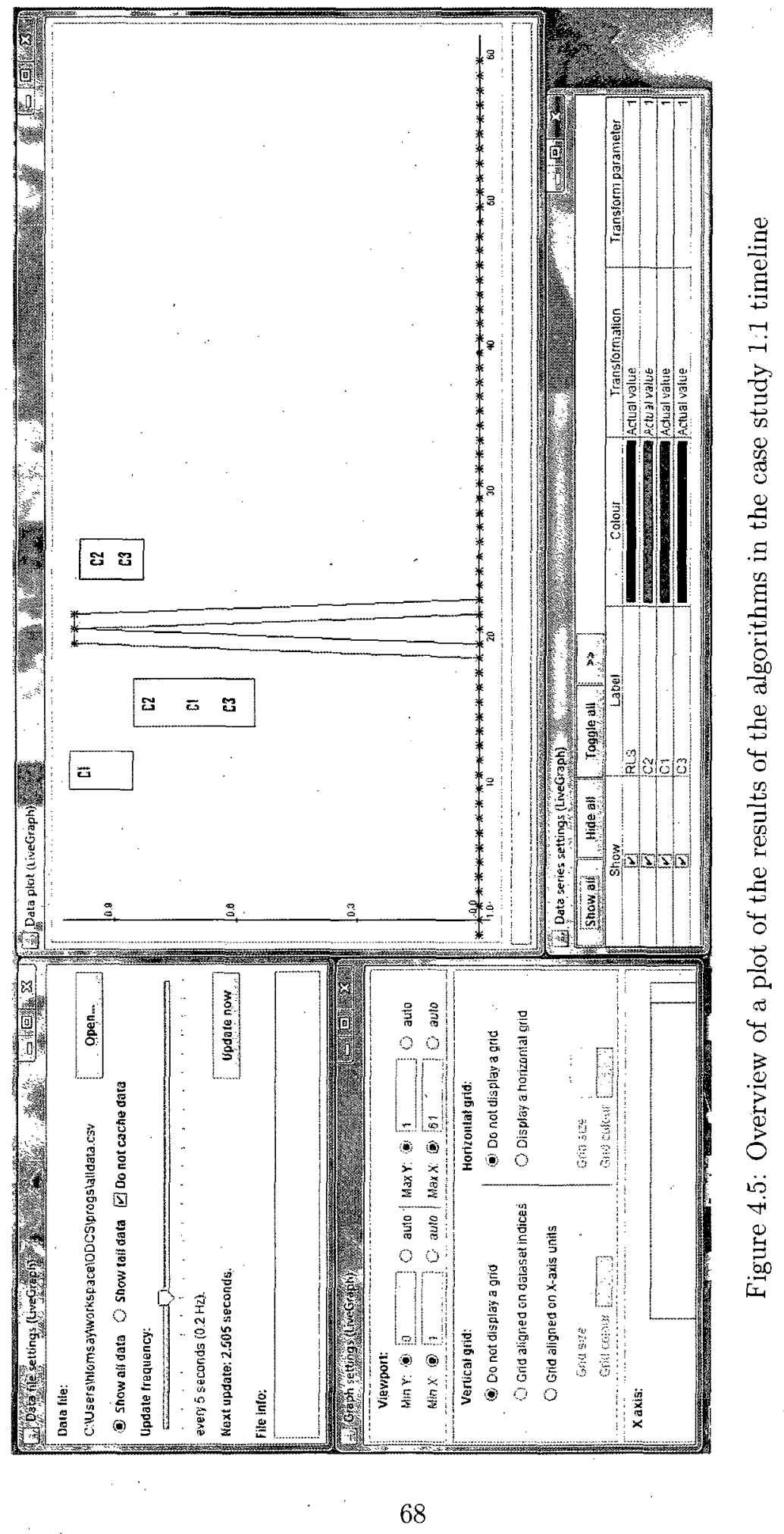




\begin{tabular}{|c|c|c|c|c|}
\hline Interval & Event Name & Dependency & Algorithm & Output \\
\hline \multirow{4}{*}{ Case1.1Interval0 } & Case1.1C3UEvent0 & - & C3-Ultra & c3results.txt \\
\hline & Case1.1RLSEvent1 & - & RLS & rlsresults.txt \\
\hline & Case1.1C2MEvent2 & - & C2Medium & c2results.txt \\
\hline & Case1.1C1MEvent3 & - & C1-Mild & c1results.txt \\
\hline \multirow{5}{*}{ Case1.1Interval1 } & Case1.1FilEvent4 & Case1.1C3UEvent0, & FileMerger & alldata.csv \\
\hline & & Case1.1RLSEvent1, & & \\
\hline & & Case1.1C2MEvent2, & & \\
\hline & & Case1.1C1MEvent3 & & \\
\hline & Case1.1LivEvent5 & Case1.1FilEvent 4 & LiveGraph & graph plot \\
\hline
\end{tabular}

Table 4.2: The created events in case study 1.1

\begin{tabular}{|c|c|c|c|c|}
\hline Interval & Event Name & Dependency & Algorithm & Output \\
\hline \multirow{3}{*}{ Case2Interval0 } & Case2C3UEvent0 & - & C3-Ultra & c3results.txt \\
\hline & Case2C2MEvent1 & - & C2Medium & c2results.txt \\
\hline & Case2C1MEvent2 & - & C1-Mild & c1results.txt \\
\hline \multirow[t]{2}{*}{ Case2Interval1 } & Case2FilEvent3 & $\begin{array}{l}\text { Case2C3UEvent0, } \\
\text { Case2C2MEvent1, }\end{array}$ & FileMerger & alldata.csv \\
\hline & Case2MajEvent 4 & $\begin{array}{l}\text { Case2C1MEvent2 } \\
\text { Case2FilEvent3 }\end{array}$ & MajorityVote & Voteresults.txt \\
\hline
\end{tabular}

Table 4.3: The created events in case study 2

(C1, C2 and C3). The algorithms are each connected to the FileMerger module and run. However, instead of employing a graphing tool a voting module that takes a vote on the alerts produced by the participant algorithms is being used. The voting algorithm used in this case study is a custom made majority vote that signals an alert only when the majority of the algorithms indicated it to be. The output of the vote algorithm can then be graphed or used for any other purpose. The case study's events and output are summarized in table 4.3 and Figure 4.6. 


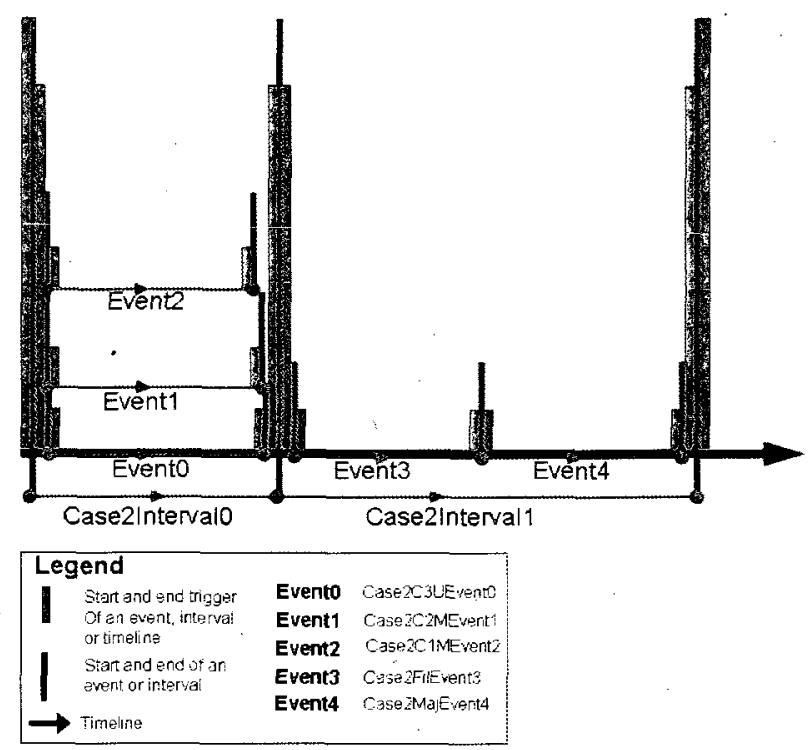

Figure 4.6: Overview of case study 2 timeline

\subsubsection{Case Study 3: Automated Use of the Architectural Elements}

Earlier on we mentioned that the architectural elements are the components that provide the gluing". functionality. These are the components that enable composition by facilitating connectivity between algorithms. In this case study trivial programs are used to demonstrate the functions of this elements and how the system automates their use. Two programs are used; dummyFileWrite and genBool. The program dummyFileWrite generates a series of numbers and writes them to a file, while genBool takes in input through the standard input stream, determines if its greater than a preset number and prints to the output stream either a true or false (true if it is greater and false if it is less). These two are connected to each other with dummyFile Write being the sending party. It is fairly noticeable though that the streams of the nodes being connected are not compatible therefore there is a need to 

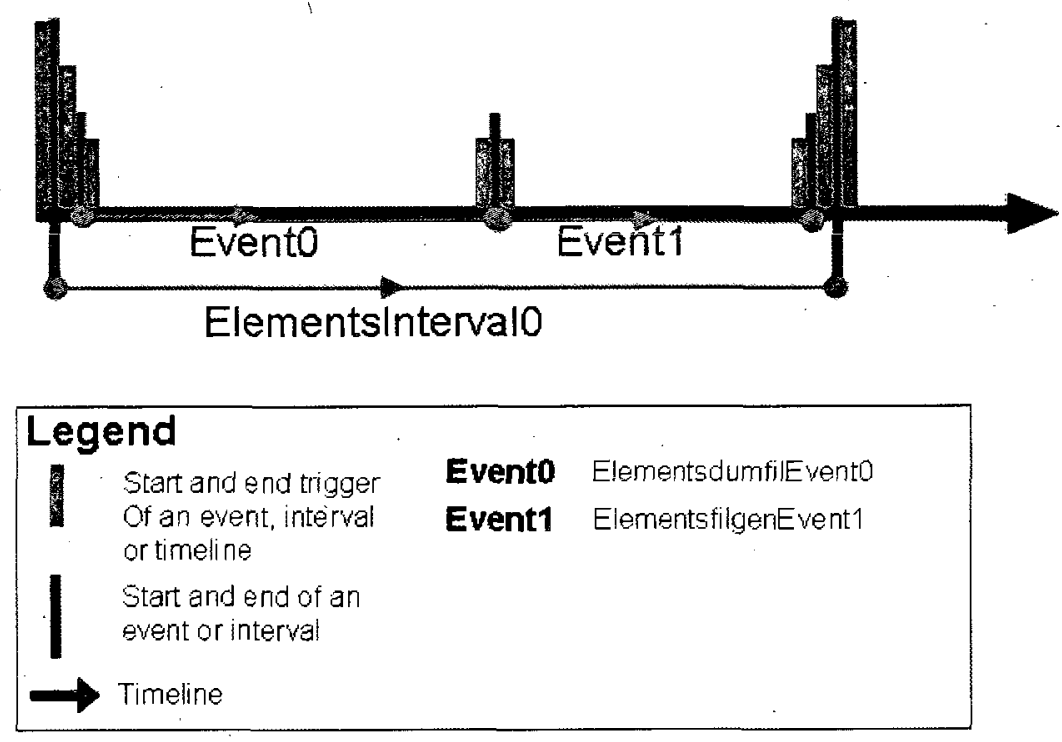

Figure 4.7: Demonstration of the use of the architectural elements

provide a bridge between the two. The system then detects this incompatibility while trying to connect the two algorithms, determines and searches for the appropriate element to bridge this incompatibility and inserts a new Task between the two. This task is another program FileToStdout whose sole purpose is to read data from the file produced by the sending program and pipes its output to the intended recipient program. The timeline resulting from this has two events; ElementsdumfilEvent0dummyFileWrite writes numbers to the file, and ElementsfilgenEvent1-File ToStdout reads the file and iteratively pipes the numbers to genBool. Figure 4.7 and table 4.4 shows this timeline. 


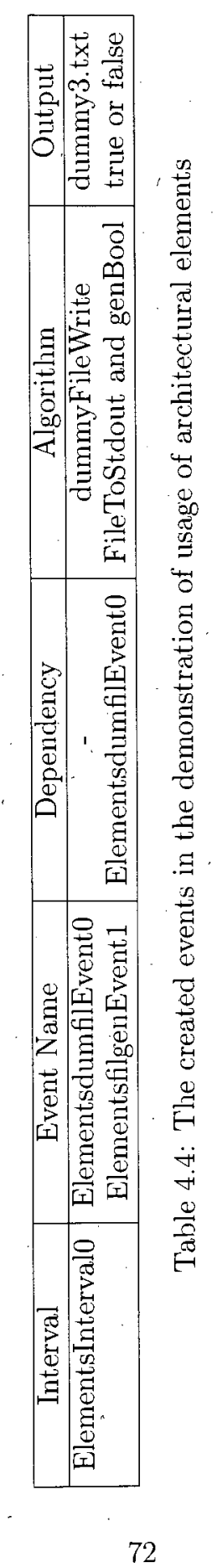




\begin{tabular}{|c|c|c|}
\hline Event & Start Time & End Time \\
\hline Case1RevRLSEvent0 & $21: 50: 56$ & $21: 50: 56$ \\
Case1RevC3UEvent1 & $21: 50: 56$ & $21: 50: 56$ \\
Case1RevC2MEvent2 & $21: 50: 56$ & $21: 50: 56$ \\
Case1RevC1MEvent3 & $21: 50: 56$ & $21: 50: 56$ \\
\hline
\end{tabular}

Table 4.5: Concurrency times with the actual Syndromic Surveillance algorithms (Case Study 1)

\begin{tabular}{|c|c|c|}
\hline Event & Start Time & End Time \\
\hline ConcurrencyDelay2DumEvent0 & $20: 15: 23$ & $20: 15: 33$ \\
ConcurrencyDelay2DumEvent1 & $20: 15: 23$ & $20: 15: 43$ \\
ConcurrencyDelay2DumEvent2 & $20: 15: 23$ & $20: 15: 33$ \\
\hline
\end{tabular}

Table 4.6: Concurrency with dummy programs that have delays on them

\subsubsection{Concurrency test}

Ascertaining that indeed the events run concurrently (in case studies where concurrency waś claimed) was a bit of a challenge. This is because the events completed very fast and it was therefore difficult to tell the difference between those that ran concurrently and those that ran sequentially. This, is equally applicable to all the case studies and algorithms with the exception of LiveGraph which runs until physically terminated by the user. Table 4.5 shows the execution times of the events in case study 1. A test case study was then devised to determine if there was concurrency. Three test programs were written with delays in them so that overlaps and time difference can be significant. This is similar to case study 1. The times from this test are detailed in table 4.6 .

From table 4.5 we could seè that all events executed and completed within a second and it is actually difficult to tell if one of the events had to wait for the other to complete or none had to wait. Table 4.6 on the other hand has visible time differences 
and the overlaps are significant. We can see that all events were started at the same time, however, ConcurrencyDelay2DumEventO and ConcurrencyDelay2DumEvent2 completed after 10 seconds while ConcurrencyDelay2DumEvent1 continued on for 10 more seconds ${ }^{1}$. The sequencing of the events is more clearer here than it was in case study 1 . We therefore can ascertain that the events ran concurrent to each other.

\footnotetext{
${ }^{1}$ Events need not have started at exactly the same time.
} 


\section{Chapter 5}

\section{Analysis}

The previous chapter presented several case studies to illustrate the system composition, architectural, and software quality aspects of an Ontology driven prototype system. It also provided basic definitions that would be followed in this chapter. Chapter 3 detailed the Ontology driven approach to system composition by discussing the development of the prototype system and the components it is comprised of. In this analysis we reflect on these chapters by discussing the benefits of such an approach through careful analysis of the case studies presented in the previous chapter in relation to the three aspects of the system mentioned earlier. More focus will be placed on the software quality factors since they relate to and are affected by the other two. The intention, therefore, is to identify the aspects of the approach that address these factors and how they do so. This analysis also highlights some of the limitations of the approach. 


\subsection{Reusability and the Aspects of the Approach that}

\section{Addresses it}

Reusability was defined in section 4.1 .3 as the extent to which a program can be used in other program. Thus our focus for this quality attribute is on how our design approach supports this concept. The thrust of this approach is in the use of algorithms as the fundamental units that provide a useful function. These algorithms are already existing self contained software programs that were written for a specific purpose. These can then be pooled into an organized unit to serve a specific purpose therefore reusing what already exists.

Each of the case studies described in chapter 4 reused algorithms that were designed for other purposes in specific domanis. The breadth of the algorithms' purpose, nature, implementation, and technology is very diverse ranging from simple custom made algorithms to open source programs. Consider the algorithms used in case study 1 ; these are all statistical algorithms written in the $\mathrm{C}$ programming language to perform outbreak detection. Although they are borrowed implementations of functionality present in other systems, they were however, never designed with this system in mind but were rather plugged-in to provide the detection functionality. The concept of reusability is even clearer in case study 1.1 where in addition to the algorithms described in case study 1 an open source and already packaged program called LiveGraph is added to the mix to provide the visualization functionality by plotting

the results of the algorithms into a graph. This then renders the idea of creating a program specifically for this system redundant. Time and possibly costs associated 
with their development is therefore saved. Duplication of work is also avoided as the creators of these programs have in essence contributed to the pool of algorithms ready for inclusion into a system.

Ontologies The main aspect of this approach that makes reusability of existing programs possible is the use of Ontologies. All of the algorithms being used in the case studies are described in an algorithm Ontology thereby explicitly exposing what is needed to use a particular algorithm without concern of its implementation details.

Modular Contribution Discussed earlier is the notion that algorithms are self contained programs that can be run. What the approach advocates for then is a process based platform for the use of these pieces of code. What you get then is a collection of libraries, and applications that one can make use of. This can be likened to the open source model where code can be contributed and reused provided one conforms to predefined standards. This then opens up for a discussion of two rather antagonistic concepts; centralized control and distributed processing. The Ontologies provides a single point for manipulation of the functionality of the algorithms by alteration of the parameters that controls the overall operation of the algorithms. This is because the descriptions of the algorithms or programs reside in an Ontology and can be modified from there. However the location of the actual algorithms and where they are run need not be in a central location hence distributed operation. Even though not seriously explored in this thesis, technologies exists that make distribution of execution a relatively trivial process compared to a few years ago such as multi- 
threaded processors, multiple core processors, grid computing etc.

The advantages of this process-based approach are:

- Use of legacy code

Some domains still use legacy systems and may be very reluctant to migrate their code to object oriented languages for example. However, the algorithms may be deemed useful by the user composing a system. In this process based approach the algorithm can be used and run in its native environment with less effort.

- Use of different programming languages and technologies

With other approaches, to use an algorithm or program written in a different programming language there is a need to use wrappers, provided there is a wrapper for the particular languages ${ }^{1}$. Here the algorithm is run as a process and its results are grabbed and used.

- Versioning

System components change over time. This may be necessitated by changes in user needs, discovery of a better way to solve the problem, change in project scope etc. System components (algorithm and architectural elements) are seen in this approach as self-contained elements with implementation details encapsulated. Therefore when a new version of the element is made available, switching to this new algorithm is simplified as the user will just point the system to the

\footnotetext{
${ }^{1}$ This maybe trivial since most languages do have wrappers to use code written in other languages even though that availability is dependant upon the generosity of someone within the user community to write such a wrapper.
} 
new version of the algorithm.

- System Neutral

We are not claiming that our system will deploy and provide every environment needed for every component available. However, the ontologies we have can be extended to allow for the system to provide a diagnosis of the current environment for suitability to the algorithms to be used. The approach is platform neutral in that it was not written to run on one type of platform and the components could also be run in their native environment.

Some of the limitations of this process-based approach are:

- Critical mass

This refers to having enough known algorithms to make this approach viable.

- Trust

Trust is an issue especially that the model adopted is reliant on contributions by different people.

- Performance

We foresee that there maybe an impact on performance when following this model, however, its relevance in our context is questionable. Please see a detailed discussion on performance in section 5.4.3.

please refer to section 5.4 for detailed discussions of the limitations 


\subsection{Flexibility and the Aspects of the Approach that}

\section{Addresses it}

In section 4.1.3 we defined flexibility as the effort needed to change a functional program, therefore the context of the discussion for this quality attribute is ease of modification and extension of a composed system. The ability to run an algorithm that was made for a particular purpose provides a specific functionality to the user. However, having the ability to make use of more than one algorithms for the same purpose provides added value. This is especially true for domains like syndromic surveillance where decisions are reliant on alerts produced by detection systems. Consider case study 1 described in section 4.2 .3 for example; more weight is placed on the epidemiologist's reason to believe that there is an outbreak if two of the three algorithms signals an outbreak occurrence rather than just one. That belief is further amplified should all three algorithms sound an alert. The ability to have varying algorithms agreeing or disagreeing on a subject matter provide the users with more information to work with when making decisions on the actions to take. This flexibility also allows for other important factors such as the verification of the correctness of the composed system, see discussion on testability in section 5.3.

The idea of non-programming skill dependant system development does not only seem appealing but it is rather demonstrated to be plausible. The case studies presented in chapter 4 illustrated to this effect. In each case study algorithms were . picked for inclusion into a system and a specific systematic ordering of the algorithms was done to complete the system. The decision to include or exclude a certain algo- 
rithm lies with the user who in this case is also the designer of the system. Contrast this to a system that ships with a set of predefined algorithms which may not necessarily suite the purpose of the user. Although this approach may have its own perks it is rather rigid. Even for those designed with extensibility in mind the actual process of extending the system is heavily reliant on the availability of skilled personnel.

In the proposed approach the user is not a passive entity to whom things are imposed. The approach is user-centric in that they are the ones driving the composition of the system. The ontologies makes adding and removing algorithm an easy process thus allowing a user to "experiment" with their system design in a much larger sense since they can add programs that were never intended for their domain. Take for example adding an artificial neural network algorithm (ANN) such as support vector machine (SVM) to a syndromic surveillance system to classify data - the SVM is a well known technique in the ANN community and in the data analysis community but is probably not that familiar to epidemiologists. Thus new methodologies and techíiques can be more easily migrated into an application domain by the users themselves. The user through this exploration gains more understanding of the software/techniques being used rather than perceiving everything to be a blackbox that only experts can explain. It must be noted though that this may not be for all users but for those who desires to explore and venture into unknown territories that may eventually pay dividend (coming up with a solution to a big problem they may have). 
Ontologies What the Ontologies offer in this approach is the ability to expose the description of the candidate algorithms for composition. Possibilities that would have otherwise been difficult are simplified by making transparent the knowledge about the aspects of the system. The ease of creation and modification of a system is reduced to a few mouse clicks and a series of keys strokes even by what may be termed laymen. The class structure of the system itself matches the structure of the Ontologies thereby making replication of ontological instances into class objects seamless. Therefore manipulation and reuse of this objects is also simplified. Having knowledge of what each algorithm takes in as input and the output it produces avails the possibility for construction of complex systems through connection of the algorithms. Leading to useful combinations that targets specific problems. It also provides future capability to automate this process for the user. This automation, however, is reliant on the availability of more algorithms and architectural elements. One form of automation has already been demonstrated as detailed in case study 4.2 .5 . The user gets exposed to the available algorithms, selects the algorithms of choice and then the system adds architectural elements, testing functions, error. handling, concurrency, etc.

Presented in this thesis is also an Ontology for the description of the order of events execution. This Ontology also opens up thrilling possibilities in as far as creation of applications. Consider the timelines for the case studies discussed in the previous chapter; figure 4.3 through 4.6 and their associated tables. The timeline can be as simple as timeline 4.3 with one interval that has three events executing concurrently or as.somewhat complex as timeline 4.4 that defines two intervals with 
four events and two events, respectively. Each of the events could be making use of several algorithm performing a variety of tasks. The order of the event execution need not be linear like the ones presented in the case studies. The Ontology provides the flexibility to have other complex arrangements such as branching where the next event to execute may be one event rather than the other depending on the results of the preceding event or some other condition. Concurrency is not always a part of packaged systems and thus this arrangement that allows for concurrency as an option when appropriate and possible is an advantage. Once again this can be automated in the future for the user.

Suppose a user is interested in knowing when in a given time frame there was an outbreak alert, irrespective of which algorithm signalled the alert. This may be necessary in a situation where the outbreak in question is very infectious with high fatality rate therefore requiring immediate response. The user may opt to use an arrangement depicted in figure 5.1. In this case data for a particular day is passed to the first algorithm C1. When there is an alert a plot is made on the graph otherwise the same data is sent to another algorithm C2 which checks for an occurrence, if there is one then a plot is made otherwise C3 is invoked which repeats the whole process. This recurs until all days are checked and plotted.

Architecture-centric Architectures and architectural elements are the enablers of the building of applications from already existing algorithms. These are the components underlying the data flow within the composed system. These elements coupled with ontological descriptions offer connectivity for disjointed algorithms enabling con- 


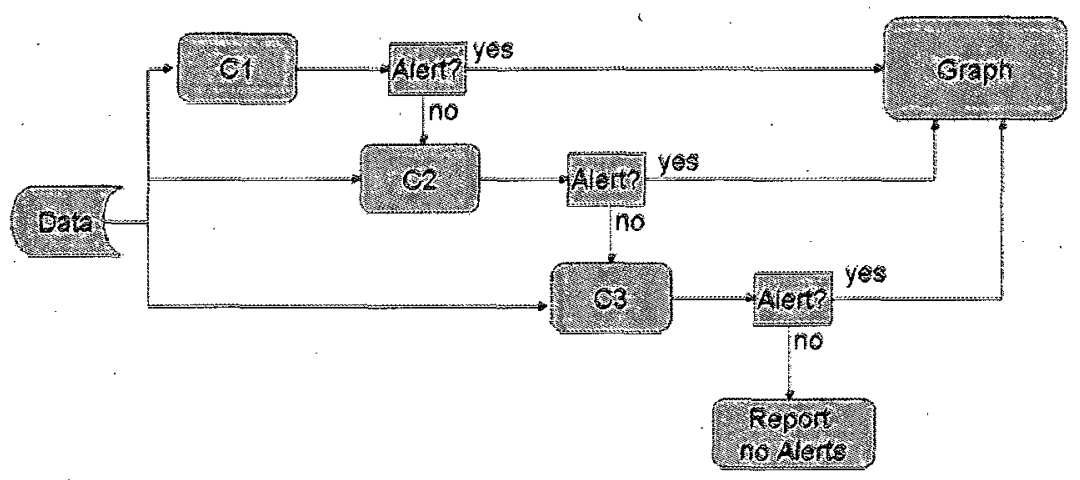

Figure 5.1: Overview of a possible branch arrangement

struction of systems that can be extend and modified with ease. An approach that has a focus in describing and building from these base elements is deemed to be ideal.

As mentioned earlier the appeal and overall usefulness of this approach is dependant on the availability of the algorithms and architectural elements. The problem facing web services maybe be eminent here as well in that there maybe no real incentives for developers to create and avail these algorithms and elements. The actual programming may not be that difficult depending on the level of skill. Creation of a substantial number of these elements may start of as directives from domain experts who may find this useful and therefore engage skilled personnel to make the algorithms they want. The algorithms to be developed could range from domain specific algorithms such as image processing algorithms, signal processing to general purpose algorithms including error checking modules, testing modules. Architectural elements such as those that facilitate distributed execution (e.g. sockets) will also need to be incorporated to make this attractive and useful to a wide audience of users. 


\subsection{Testability}

The correctness of a built system needs to be established. There are several ways in which this can be done, let us consider two of these; testing the accuracy of each constituent algorithm and testing the overall correctness of the system. Earlier we mentioned the notion that the modularity and descriptive nature of this approach allows for modules or algorithms to be added when needed. In syndromic surveillance there are a set of standard measures used to determine the accuracy of a system; false positive, false negatives, specificity, and sensitivity. Therefore having data that has known true outbreaks allows for these measures to be applied to individual algorithms to determine how they fair against each measure. All that has to be done is create a module that calculates these measures based on comparisons of the results of a successful execution of the constituent algorithms. This can be as simple as a slight modification of case study 1 by adding another algorithm/module (accuracy test module) that receives variable number of inputs one of which would be the true data and printing the aforementioned measures. The same can be done with case study 2 where the results of the vote can be passed to the test module along with baseline data. Another valuable option would be to have the results of all algorithms involved in the vote, the results of the vote and the baseline passed to this module for more extensive comparisons. This can reveal valuable information about how each algorithm's result fair against the vote, against each other, against the baseline data and how the vote fairs against the baseline data. More and even better test modules can be built and plugged in provided they are well described within the Ontology. 
Even though there are different types of testing, testing of individual algorithms has an impact on trust. If the algorithm is found to be correct most of the time it is used then the level of trust for that particular algorithm rises (in terms of accuracy). However, if its accuracy fluctuates or is found to be inaccurate often then trust levels will drop for that algorithm.

\subsection{Limitations of the Approach}

\subsubsection{Critical Mass}

Consider the object oriented paradigm; it seemed a very good idea at conception but it was not until there were enough class libraries written that it took off and its full potential was experienced. A similar situation exists within the web service paradigm where the idea seems great but there are not many useful services for the majority of the user community partly because there are few incentives for people to develop these web services. The usefulness of this prototype will also be affected by the availability of more algorithms and architectural elements.

\subsubsection{Trust}

Implementations that rely on contributions from many sources are almost always faced with questions and doubts on their trustworthiness. This implementation is no exception. While efforts in different domains are being made such as establishing trust policies in the web service domain, trust issues still persist. In retrospect, we believe Ontologies can have a significant role in handling the issue of trust. While the 
ultimate decision to trust a particular algorithm lies with the human user, Ontologies can be used to describe and avail knowledge needed to facilitate for this decision. As an example information about the authors/vendors of a particular algorithm, their background, their affiliations, etc. can be published and used to decide on the level of trust. Having said that, what we are proposing is more like the open source model in that people always use open source software contributed by other people therefore if one has problems trusting open source software they would have problems trusting this system. It must be noted though that we are not solving for the trust problem at least for the time being.

\subsubsection{Performance}

While performance was not the main focus of this thesis it however, still remains an important quality attribute to be looked at. Considering that this is a processed-based implementation that starts processes whenever an event is started, this can have significant effects on performance. Having said that, the use of multithreaded processors and distributed implementations may be explored to mitigate for this limitations.

While we concede that performance maybe slow in this approach, it may not be as relevant in our context (debatable). Our context is more on the user asking questions like "How can I design a system that meets my needs using this prototype system?" and not so much "Will a system I developed using this system be faster than an alternative approach?". In any case other approaches do not necessarily guarantee 
better performance. Without starting a lot of controversies, better performance may also be programmer dependent and not so much on the approach itself. In the event that a module was found to be slow then it may be replaced by a faster one (e.g. going from Python module to $\mathrm{C}++$ module written by an expert in optimization): 


\section{Chapter 6}

\section{Conclusions and Future Work}

\subsection{Conclusions}

In the motivation of this research we highlighted the problems of conventionally developed systems with predefined data sets and algorithms that are preset for a particular purpose. We highlighted that these systems may not be suitable for dynamic domains whose environment, parameters and needs change rapidly. We then explored how an Ontology driven approach to software composition can be used to drive the creation of adaptive systems. This was done by scrutinizing the aspect of system composition where a system is designed by simply making use of already existing algorithms and ordering them to fit a specific purpose. We covered such issues as ease of creation and extension of a system in as far as inclusion of algorithms, exclusion of algorithms, manipulation or changing the parameters that affect the overall functionality of the algorithms and support or use of certain architectures that deals with the data flow between algorithms that may ultimately affect the systems output.

For demonstration, ODCS-the prototype system, was designed and developed using the Java programming language driven by two main Ontologies; an algorithms Ontology and an execution timeline Ontology. These Ontologies describe 
the fundamental components of the system (algorithms) and the order in which the events of the system are executed, respectively. Since the Ontologies were known beforehand they were used to model the behavior of the system.

Through a careful review of a selection of case studies that illustrate the attributes of the prototype system and a critical analysis of the aspects of the system with respect to the flexibility, reusability and testability quality factors, we found an Ontology driven approach to system composition to be very flexible and easily extensible. This flexibility was facilitated by the Ontologies which exposed the description of the candidate algorithms allowing them to be included and excluded from the system with ease. A system such as ODCS could make a world of specialist algorithms and techniques available to a much wider range of domains and users than is now possible. This is because leveraging and reuse of already existing algorithms is supported since the development of a system is solely based on already existing algorithms that were contributed by several creators. The facets of the prototype system such as the Ontologies and frameworks presents other possibilities as automatic testing of the composed system. This is especially true when it comes to checking the correctness of the constituent algorithms as discussed in section 5.3. We therefore arrive at the conclusion that an Ontology driven compositional system is suitable for dynamic application areas that require a user-centric and rapid application development. 


\subsection{Future Work}

\subsubsection{Population of the Algorithm Ontology}

The algorithm Ontology used for the prototype system is not well populated with instances but rather only a few algorithms useful to the syndromic surveillance domain were described for the sole purpose of demonstration. It will therefore be important to populate the Ontology with more algorithms thereby widening the usefulness of the Ontology.

\subsubsection{Ontology Evolution}

Knowledge is non-static therefore at some point it will change. This means that the fundamental aspects which the Ontologies model and represent evolve over time. Since the building of the system is driven by the knowledge then a considerable amount of effort should be invested in investigating the level of impact this would have on the system and other systems that rely on this knowledge. This implies research into Ontology evolution strategies. This may be handy strategies that may help in mitigating the impact of changing Ontologies used as the core of systems. One example may be to partition the Ontology into static and non-static components and thereby deciding on which parts of the Ontology may be changed and which parts may not be changed. This coupled with how the changes are propagated to all systems using the Ontology as well as decisions on the roles each person/system plays in the management of the Ontology makes a necessary research subject. 


\subsubsection{Integration of Existing Ontologies}

While the Ontologies described in this thesis serve a good purpose of demonstrating the concept of Ontology driven system composition, automating the functions of the system may require additional descriptions. Existing Ontologies could be employed in this regard. One such Ontology is the data source Ontology developed at Stanford University for use in a system called BIOSTORM [7]. This is a rather comprehensive Ontology that offers flexible descriptions of the inputs of the system. Therefore as a possible future extension of the current Ontologies, this Ontology could be integrated and used.

\subsubsection{Architectures and Design Patterns}

In his masters degree thesis, Chruszcz [6] suggests explicit description of design patterns in the form of Ontologies. These are documented solutions that have recurred in many problems [32]. Since design patterns have proven themselves (through recurrence) to be good solutions, their use will help streamline the development process. While we share Chruszcz's thoughts, we never got to provide these descriptions and hence this remains the logical next step. Such patterns as pipeline also known as pipes and filters, publish-subscribe (including its subsets such as the observer design pattern) could be defined in the Ontologies to describe the flow of data. 


\subsubsection{Graphical User Interface}

Graphical user interfaces offer an intuitive means to control a system and asses its state provided they are properly designed. The prototype ODCS described in this thesis does not yet include a user interface to allow for simple manipulation of the Ontologies by a non-technical domain expert. The interface is by design left as a detachable module since it should be custom designed to fit the abilities, expectations and conceptual framework of the user's domain of expertise. While the interface may change, the underlying algorithms and architectures captured in the Ontologies are available for use by multiple domains. This will allow many application areas to share algorithms developed for different domains. For example, there are many pattern recognition and data mining algorithms developed by the machine intelligence community that are often overlooked by users in domains such as epidemiology, economics, etc., and thus their applications are unnecessarily limited.

An ideal user interface for this type of system would be one that takes into consideration the needs of three types of users; the authors and contributors of the algorithms, the domain experts, and the designers of the system. The user interface should provide.an easy way for algorithm contributors to add algorithms by creating instances of the algorithm Ontology and defining relevant properties for

the algorithms they are publishing. It also should offer a means and rules of deleting and modifying instances. For the domain experts the interface should provide a way to query the Ontology for desired algorithms, select the algorithms for use and order them in a way desirable to them. The domain experts should have a way of providing 
needed input and viewing the output of running their system. The designers of the system will also need to perform administrative tasks.

\subsubsection{Verification and Validation}

Building a system is one aspect, however, ascertaining the correctness and proper functioning of the built system is paramount. Automating these for the user is desirable and hence future exploration of how Ontologies can be used to do this automation would add value to the functioning of the proposed prototype. Such concepts as error checking, load balancing and deadlock detection are worth looking at. We laid some ground work for error checking in our description of the timeline Ontology, however, work in this regard needs more attention. Considering that this is a processed-based implementation that starts processes whenever an event is started, this can have significant effects on performance. Therefore future work geared towards extending this system to include the concept of load balancing would be a worthwhile investment. With the use of data flow architectures such as pipelining, possibilities of deadlocks are eminent. Therefore appropriate measures are needed to detect and mitigate for these possibilities. A deadlock could arise from such situations as a dependant algorithm waiting endlessly for input from a sending algorithm that never provides the needed input.

The plausibility of the prototype system was demonstrated through the case studies in chapter 4 and the detailed discussions in the analysis chapter. However, with these proposed extensions in place this prototype system would be attractive and useful to 
a larger user audience.

$\checkmark$ 


\section{Appendix A}

\section{Overview of the Ontologies}

\section{A.1 Overview of the Algorithm Ontology}

In this section, a complete structure of the algorithm Ontology used for the development of ODCS is presented. As it may be true for most Ontologies the Thing class is the super class of all classes in an Ontology. Figure A.1 depicts the hierarchy of this class.

\begin{tabular}{|c|c|}
\hline \multicolumn{2}{|l|}{8 shithens } \\
\hline \multicolumn{2}{|l|}{ 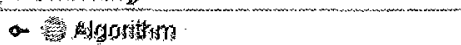 } \\
\hline \multicolumn{2}{|l|}{ - Asyoritrirrile } \\
\hline \multicolumn{2}{|l|}{ Ostakgo } \\
\hline \multicolumn{2}{|l|}{ 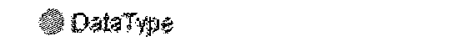 } \\
\hline$\infty$ then ind & \\
\hline 触 Location & \\
\hline 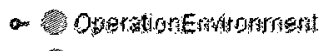 & \\
\hline 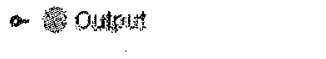 & \\
\hline
\end{tabular}

Figure A.1: Overview of the Thing Class Hierarchy (immediate children)

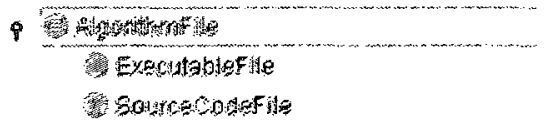

Figure A.2: Overview of the AlgorithmFile Class Hierarchy 


\begin{tabular}{|c|c|c|}
\hline \multicolumn{3}{|c|}{ 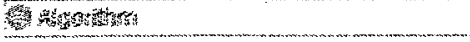 } \\
\hline \multicolumn{3}{|c|}{ P WustuterfuraiElements } \\
\hline & Yanonter & \\
\hline & 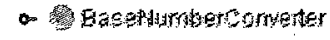 & \\
\hline & 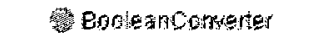 & \\
\hline & 整 Chatcorsterter & \\
\hline & 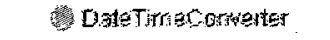 & \\
\hline & 4y stringormenter & \\
\hline & Fidekdanager & \\
\hline & Whate & \\
\hline \multicolumn{3}{|c|}{ 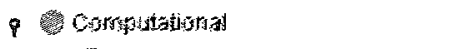 } \\
\hline \multicolumn{3}{|c|}{ Thenerapuraso } \\
\hline \multicolumn{3}{|c|}{ Granploding } \\
\hline \multicolumn{3}{|c|}{ Shang Foreseing } \\
\hline \multicolumn{3}{|c|}{ 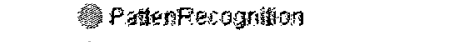 } \\
\hline \multicolumn{3}{|c|}{ 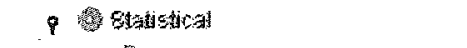 } \\
\hline \multicolumn{3}{|c|}{ Toven } \\
\hline & 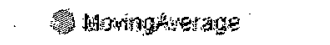 & \\
\hline & 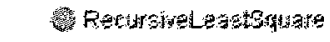 & \\
\hline
\end{tabular}

Figure A.3: Overview of the Algorithm Class Hierarchy

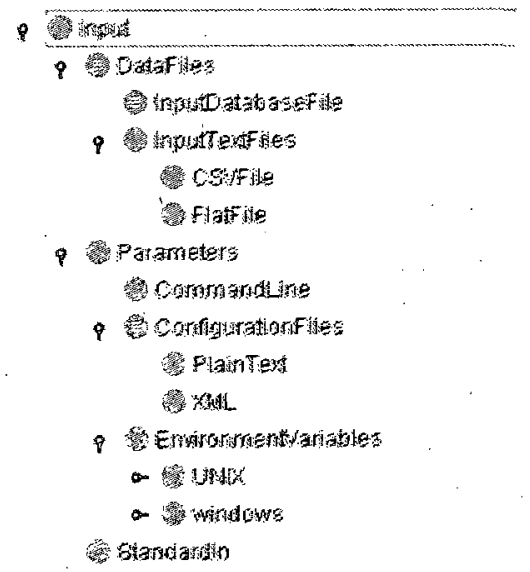

Figure A.4: Overview of the Input Class Hierarchy 


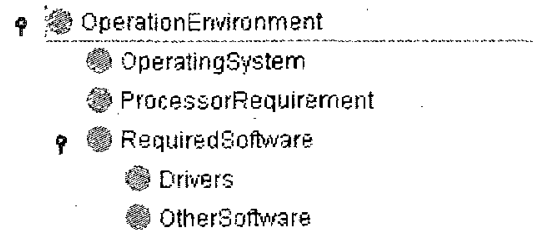

Figure A.5: Overview of the OperationEnvironment Class Hierarchy

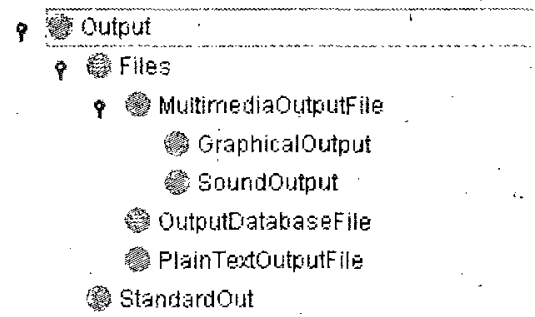

Figure A.6: Overview of the Output Class Hierarchy 


\section{A.2 Overview of the Execution Timeline Ontology}

This sections presents an overview of the ExecutionTimeline Ontology. Once again all classes in this Ontology are subclasses of the Thing class. The class hierarchy of this Ontology is depicted by figure A.7.

\begin{tabular}{|c|c|}
\hline & 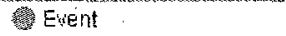 \\
\hline & 叠 ExeculioriError \\
\hline & 筑 inteval \\
\hline & 繁 Timeline \\
\hline 8 & Wrigger \\
\hline & WheckfleExist \\
\hline & Wheckinputsuailable \\
\hline & FuriNextFrocess \\
\hline & Terminateprocess \\
\hline
\end{tabular}

Figure A.7: Overview of the ExecutionTimeline Ontology Class Hierarchy 


\section{Bibliography}

[1] M.B. Blake and M.F. Nowlan. Taming web services from the wild. IEEE Internet Computing, 12(5):62-69, 2008.

[2] B.W. Boehm, J.R. Brown, H. Kaspar, M. Lipow, G.J. Macleod, and M.J. Merrit. Characteristics of Software Quality. North-Holland Pub. Co, 1978.

[3] H.S. Burkom. Development, Adaptation, and Assessment of Arleting Algorithms for Biosurveillance. Johns Hopkins APL Technical Digest, 24(4), 2003.

[4] J.P. Cavano and J.A. McCall. A framework for the measurement of software quality. In Proceedings of the software quality assurance workshop on functional and performance issues, pages 133-139, 1978.

[5] A. Charfi and M. Mezini. AO4BPEL: An Aspect-oriented Extension to BPEL. World Wide Web, 10(3):309-344, 2007.

[6] B. Chruszcz. Extensibility in semantic web description of syndromic surveillance systems. Master's thesis, University of Guelph Canada, 2008.

[7] M. Crubezy, M. O Connor, Z. Pincus, M.A. Musen, and D.L. Buckeridge. Ontology-centered syndromic surveillance for bioterrorism. IEEE Intelligent Systems, 20(3):26-35, 2005.

[8] G. Diallo, P. Kostkova, G. Jawaheer, S. Jupp, and R. Stevens. Process of building a vocabulary for the infection domain. In 21st IEEE International Symposium on Computer-Based Medical Systems, pages 308-313, 2008.

[9] T.S. Dillon, E. Chang, and M. Hadzic. Ontology support for biomedical information resources. In 21st IEEE International Symposium on Computer-Based Medical Systems, pages 7-16, 2008.

[10] J.S Dong. Software modeling techniques and the semantic web. In Proceedings of the 26th International Conference on Software Engineering, pages 1160-1163, 2004 .

[11] R.W. Feenstra, M. Janssen, and R.W. Wagenaar. Evaluating web composition methods: The need for including multi-actor elements. The Electronic Journal of E-Government, 15(2):153-164, 2007.

[12. G.R. Guthrie. Detection of disease outbreaks in pharmaceutical sales: An investigation of an artificial neural network approach. Master's thesis, University of Guelph Canada, 2005. 
[13] M. Hadzic and E. Chang. Ontology-based support for human disease study. In Proceedings of the 38th Annual Hawaii International Conference on System Sciences, pages 1-7, 2005.

[14] K.J. Henning. Overview of syndromic surveillance-what is syndromic surveillance? $M M W R, 53: 5-11,2004$.

[15] H. Hlomani and D. A. Stacey. An ontology driven approach to software systems composition. In International Conference on Knowledge Engineering and Ontology Development, 2009.

[16] M. Horridge, S. Jupp, G. Moulton, A. Rector, R. Stevens, and C. Wroe. A Practical Guide to Build OWL Ontologies Using the Protg-OWL Plug-in COODE Tools Edition 1.0, 1.1 edition, 2007. Retrieved June 25, 2009, from http://www.co-ode.org/resources/tutorials/ProtegeOWLTutorial-p4.0.pdf.

[17] L. Hutwagner, W. Thompson, G.M. Seeman, and T. Treadwell. The Bioterrorism Preparedness and Response Early Aberration Reporting System(EARS). Journal of Urban Health:Bulletin of New York Academy of Medicine, 80, 2003.

[18] IEEE. IEEE Std 1061-1998, (Revision of IEEE Std 1061-1992)Standard for a Software Quality Metrics Methodology. New York: Institute of Electrical and Electronics Engineers, 1998.

[19] J.E. Gaffney Jr. Metrics in software quality assurance. In Proceedings of the $A C M$ '81 conference, pages 126-130, 1981.

[20] A. Kalyanpur, D. J. Pastor, S. Battle, and J. Padget. Automatic mapping of OWL ontologies into Java. In 16th International Conference on Software Engineering and Knowledge Engineering, pages 98-103, 2004.

[21] A: Kawazoe, H. Chanlekha, M. Shigematsu, and N. Collier. Structuring an event ontology for disease outbreak detection. BMC Bioinformatics, 9 Suppl $3: 1-8,2008$.

[22] H. Knublauch. Ontology-Driven Software Development in the Context of the Semantic Web: An Example Scenario with Protg-OWL. In International Workshop on the Model-Driven Semantic Web, 2004.

[23] W.B. Langdon and J.R. Koza. Genetic Programming and Data Structures: Genetic Programming + Data Structures = Automatic Programming! Kluwer Academic Publishers, 1998.

[24] N. Milanovic and M. Malek. Current solutions for web service composition. IEEE Internet Computing, 8(6):51-59, 2004.

[25] P. Mirhaji, S.W. Casscells, D. Allemang, and R. Coyne. Improving the public health information network through semantic modeling. IEEE Intelligent Systems, 22(3):13-17, 2007. 
[26] F. Mostashari and J.J. Hartman. Syndromic surveillance: a local perspective. Journal of Urban Health, 80:11-17, 2003.

[27] N.F. Noy, M. Sintek, S. Decker, M. Crubezy, R.W. Fergerson, and M.A. Musen. Creating semantic web contents with protege-2000. IEEE Intelligent Systems, 16(2):60-71, 2001.

[28] International Standard Organization. ISO/IEC 9126:1991, 1991.

[29] M.P. Papazoglou. Service oriented computing: Concepts, charecteristics and directions. In Fourth International Conference on Web Information Systems Engineering, pages 3-12, 2003.

[30] R.T. Plant. Factors in software quality for knowledge-based systems. Inf. Softw. Technol, 33(7):527-536, 1991.

[31] N. Shadbolt, W. Hall, and T. Berners-Lee. The semantic web revised. IEEE Intelligent Systems, 21:96-101, 2006.

[32] A. Shalloway and J. Trott. Design patterns explained: a new perspective on object-oriented design. Addison-Wesley, 2002.

[33] D.M. Sosin. Draft framework for evaluating syndromic surveillance systems. Journal of Urban Health, 80:i8-i13, 2003.

[34] W.M. Spears. Evoluttionary Algorithms: The role of mutation and recombination. Springer, 2000.

[35] Stanford Center for Biomedical Informatics Research. What is Protege-OWL?, 2009. Retrieved June 25, 2009, from http://protege.stanford.edu/overview/protege-owl.html.

[36] B. Szekely. Jastor: Typesafe, Ontology Driven RDF Access from Java, 2009. Retrieved June 25, 2009, from http://jastor.sourceforge.net/.

[37] R.N. Taylor, N. Medvidovic, and E.M. Dashofy. Software Architecture Foundations, Theory, and Practice. John Wiley and Sons Inc., 2008.

[38] A. Tjoa, A. Andjomshoaa, F. Shayeganfar, and R. Wagner. Semantic web challenges and new requirements. In Proceedings of the 16th International Workshop on Databases and Expert Systems Applications, pages 1160-1163, 2005.

[39] F. Tsui, J.U. Espino, V.M. Dato, P.H., Gesteland, J. Hutman, and M.M. Wagner. A Technical Description of RODS: A Real-time Public Health Surveillance System. Journal of the American Medical Informatics Association, 10(5):399-408, 2003.

[40] G.F. Wetzel and W.G. Bulgren. The algorithmic process-An intorduction to problem solving. Science Research Associates Inc, 1985. 
[41] M.A. Wirth. Confessions of a coding monkey-Learning the subtle art of programming. Kendall/Hunt Publishing Company, 2007.

[42] J. Zhang. Ontology and the semantic web. In Proceedings of the North American Symposium on Knowledge Organization, pages 9-20, 2007.

[43] M. Zimmermann. Owl2Java - A Java Code Generator for $O W L$, 2009. Retrieved June 25, 2009, from http://www.incunabulum.de/projects/it/owl2java/owl2java-a-owl2javagenerator. 